\title{
Sources of disconnection in neurocognitive aging: cerebral white-matter integrity, resting-state functional connectivity, and white-matter hyperintensity volume
}

\author{
David J. Madden $^{\mathrm{a}, \mathrm{b}, *}$, Emily L. Parks ${ }^{\mathrm{a}, \mathrm{b}}$, Catherine W. Tallman ${ }^{\mathrm{a}}$, Maria A. Boylan ${ }^{\mathrm{a}}$, \\ David A. Hoagey ${ }^{a}$, Sally B. Cocjin ${ }^{a}$, Lauren E. Packard ${ }^{a}$, Micah A. Johnson ${ }^{a}$, \\ Ying-hui Chou ${ }^{\mathrm{a}, \mathrm{b}}$, Guy G. Potter ${ }^{\mathrm{a}, \mathrm{b}}$, Nan-kuei Chen ${ }^{\mathrm{a}, \mathrm{c}}$, Rachel E. Siciliano ${ }^{\mathrm{a}}$, \\ Zachary A. Monge ${ }^{\mathrm{d}}$, Jesse A. Honig ${ }^{\mathrm{a}}$, Michele T. Diaz ${ }^{\mathrm{e}}$ \\ ${ }^{a}$ Brain Imaging and Analysis Center, Duke University Medical Center, Durham, NC, USA \\ ${ }^{\mathrm{b}}$ Department of Psychiatry and Behavioral Sciences, Duke University Medical Center, Durham, NC, USA \\ ${ }^{\mathrm{c}}$ Department of Radiology, Duke University Medical Center, Durham, NC, USA \\ ${ }^{\mathrm{d}}$ Center for Cognitive Neuroscience, Duke University, Durham, NC, USA \\ ${ }^{\mathrm{e}}$ Department of Psychology, Pennsylvania State University, University Park, PA, USA
}

\section{A R T I C L E I N F O}

\section{Article history:}

Received 20 May 2015

Received in revised form 5 January 2017

Accepted 6 January 2017

Keywords:

Magnetic resonance imaging

Diffusion tensor imaging

Cognition

Cortex

Mediation

\begin{abstract}
A B S T R A C T
Age-related decline in fluid cognition can be characterized as a disconnection among specific brain structures, leading to a decline in functional efficiency. The potential sources of disconnection, however, are unclear. We investigated imaging measures of cerebral white-matter integrity, resting-state functional connectivity, and white-matter hyperintensity volume as mediators of the relation between age and fluid cognition, in 145 healthy, community-dwelling adults 19-79 years of age. At a general level of analysis, with a single composite measure of fluid cognition and single measures of each of the 3 imaging modalities, age exhibited an independent influence on the cognitive and imaging measures, and the imaging variables did not mediate the age-cognition relation. At a more specific level of analysis, restingstate functional connectivity of sensorimotor networks was a significant mediator of the age-related decline in executive function. These findings suggest that different levels of analysis lead to different models of neurocognitive disconnection, and that resting-state functional connectivity, in particular, may contribute to age-related decline in executive function.
\end{abstract}

(c) 2017 Elsevier Inc. All rights reserved.
Age-related decline is frequently observed in behavioral measures of fluid cognitive ability such as elementary perceptual speed, attention, executive function, and episodic memory, whereas measures of crystallized abilities relating to knowledge and expertise exhibit little or no decline during healthy aging (Howard and Howard, 2013; Park et al., 2002; Salthouse, 1996, 2004). From neuroimaging measures, particularly magnetic resonance imaging (MRI), researchers have identified many aspects of brain structure and function that also decline during adult aging, even in the absence of frank disease (Fjell and Walhovd, 2010; Grady, 2012; Hedden and Gabrieli, 2004; Walhovd et al., 2011). A disconnection model of neurocognitive aging, building on the clinical neurological model of Geschwind (Catani and ffytche, 2005; Geschwind,

\footnotetext{
* Corresponding author at: Brain Imaging and Analysis Center, Box 3918, Duke University Medical Center, Durham, NC 27710, USA. Tel.: 919-681-9345; fax: 919681-7033.

E-mail address: david.madden@duke.edu (D.J. Madden).
}

1965a,b), is emerging as a theoretical framework for the diverse pattern of individual differences in structural and functional brain measures associated with age-related differences in cognitive functioning (Bartzokis, 2004; Bennett and Madden, 2014; Charlton et al., 2006; Fjell and Walhovd, 2010; Fjell et al., 2016; Greenwood, 2000; Madden and Parks, 2017; O'Sullivan et al., 2001; Peters, 2002; Salat, 2011). Because fluid cognition relies on the speed and efficiency of communication among large-scale neural networks, the disruption of network connections, even when not due to a specific disease, can lead to decline in cognitive performance.

A critical issue for the disconnection model is whether specific aspects of brain structure and function contribute differentially to age-related cognitive decline. In addition, this issue is relevant for clinical translation, in the context of identifying relevant biomarkers of neurocognitive function, as targets for possible intervention and rehabilitation (Bishop et al., 2010; Buckner, 2004; Jagust, 2013; Seeley et al., 2009). Hedden et al. (2016), for example, examined a wide range of structural and functional brain imaging 
measures, and behavioral measures, in 186 healthy older adults 65-90 years of age. These authors found that although a large portion of the age-related variance in cognition was shared among brain imaging measures, individual measures did exhibit unique associations with age-related decline in perceptual speed, executive function, and episodic memory. In a superset of the Hedden et al. data, analyses of resting-state functional connectivity networks indicated that frontoparietal connectivity was a mediator of the relation between other resting-state networks and cognition (Shaw et al., 2015). Fjell et al. (2016) compared multiple measures of structural and functional brain connectivity, including resting-state functional connectivity, white-matter connectivity from diffusion tensor imaging (DTI), white-matter volume, and cortical thickness, as mediators of longitudinal decline (over a 3.3 years interval), in executive function (Stroop interference). This set of imaging measures accounted for $82.5 \%$ of the variance in longitudinal decline in executive function, with the largest influence from white-matter volume.

In characterizing the sources of neurocognitive disconnection, it is important to consider alternative models of the age-related differences in brain imaging and cognitive measures. Salthouse (2011b, 2017) has pointed out that many of the findings that have been interpreted as reflecting specific mediation of age-cognition relations, by specific aspects of brain structure or function, may actually reflect the independent effects of age on the brain and on cognition. Further, it is important to consider the level of analysis as well as the alternative models. Salthouse et al. (2015) have proposed that because brain imaging measures that are treated as independent are often highly correlated, age-related effects that appear to be regionally specific may be dependent on the variance shared with other related imaging variables, which would be observable at a more general level of analysis. From factor analyses of the relation between cortical thickness (from 33 brain regions) and cognition (from 12 cognitive measures) in 297 adults between 20 and 79 years of age, Salthouse et al. proposed that many of the cortical thickness-cognition relations appeared to be operating at a broad level representing what is common among many measures and not exclusively at the level of individual factors or measures. Similarly, the pattern of age-related differences suggested independent effects of age on the cortical thickness and cognitive measures. Other analyses of this data set, however, using more regionally specific imaging measures, have identified mediation of age-related cognitive effects by localized patterns of cortical thickness (Lee et al., 2016) and white-matter integrity (Gazes et al., 2016). The Lee et al. and Gazes et al. findings raise the possibility that specific influences of brain structure and function, on agerelated differences in cognition, will be more apparent when brain imaging variables have greater anatomical specificity.

In this research, our goal was to compare 2 different models of age-related disconnection of neurocognitive function, one in which age has independent influences on the brain and fluid cognition (Salthouse, 2011b, 2017; Salthouse et al., 2015), and an alternative model in which aspects of brain structure and function are mediators of the age-cognition relation (Fjell et al., 2016; Gazes et al., 2016; Hedden et al., 2016; Lee et al., 2016). We also investigated the agerelated effects at different levels of analysis, to determine whether mediating effects are more clearly evident with regionally specific imaging measures. As an investigation of brain imaging mediators of age and cognition, this study is unique, to our knowledge, in the use of regionally specific measures from multiple imaging modalities, and multiple cognitive measures, across a wide age range of 19-79 years. We focused on 3 fluid cognitive abilities: elementary perceptual speed, executive function, and memory, which have exhibited distinguishable age-related effects in previous research (Borghesani et al., 2013; Hedden et al., 2016; Kennedy and Raz, 2009).
We chose 3 imaging modalities with established relations to adult age and fluid cognition: the integrity of normal-appearing cerebral white-matter, as measured by DTI (Bennett and Madden, 2014; Carmichael and Lockhart, 2012; Madden et al., 2012; Sullivan and Pfefferbaum, 2011), the functional connectivity of brain networks as assessed from resting-state functional MRI (fMRI; Andrews-Hanna et al., 2007; Biswal et al., 2010; Ferreira and Busatto, 2013; Shaw et al., 2015; Tomasi and Volkow, 2012), and white-matter hyperintensities (WMHs) on T2-weighted imaging, which are indicative of whitematter lesions due to cerebrovascular insufficiencies (Birdsill et al., 2014; Madden and Parks, 2017; Maillard et al., 2012; Salat, 2014a,b).

We hypothesized that different models of age-related differences in the brain imaging and cognitive measures would be apparent at different levels of analysis. At the more general level of analysis, with general fluid cognition defined from a composite of all cognitive measures, and each of the imaging modalities (white-matter integrity, resting-state functional connectivity, and WMH volume) defined as a composite measure from all of the indicator variables within the modality, we predicted that age would have independent effects on the cognitive and imaging measures. Specifically, we predicted that age-related differences in the cognitive measures would be independent of variation in the imaging measures, and that age-related differences in the imaging measures would be independent of individual differences in cognition (Salthouse, 2011b, 2017; Salthouse et al., 2015). When, in contrast, at a more finegrained level of analysis, with specific cognitive domains and regionally specific imaging measures distinguished from their shared variance, we expected that mediation of the age-cognitive relation, by the imaging variables, would be observed (Fjell et al., 2016; Gazes et al., 2016; Hedden et al., 2016; Lee et al., 2016).

Although many specific patterns of mediation are possible, in the case of white-matter integrity and resting-state functional connectivity, we were particularly interested in frontoparietal regions, in view of the previous findings implicating frontoparietal networks in age-related decline in speed and executive function (Bennett and Madden, 2014; Gold et al., 2010; Kennedy and Raz, 2009; Monge et al., 2016; Shaw et al., 2015). In the case of WMH volume, we distinguished periventricular (PV) hyperintensities from those located in deep white-matter (DWM) regions. Previous evidence suggests that PV WMHs have a relatively greater role in age-related decline in fluid cognition, by disrupting long whitematter tracts connecting cortical association regions (Smith et al., 2011; Soderlund et al., 2006; van den Heuvel et al., 2006).

\section{Materials and methods}

\subsection{Participants}

The participants were 145 healthy, community-dwelling adults (79 women) between 19 and 79 years of age recruited to be distributed relatively equally across 3 age categories: 19-39 years of age ( $n=48), 40-59$ years of age $(n=49)$, and $60-79$ years of age $(n=48)$. Participants gave written informed consent for a protocol approved by the Duke University Institutional Review Board. All participants were right-handed by self-report, completed at least 12 years of education, were free from significant health problems (including atherosclerosis, neurological, and psychiatric disorders) and were not taking medications known to affect cognitive function or cerebral blood flow (except antihypertensive agents).

During a testing session conducted on average approximately 1 month before the MRI session, participants completed several screening tests and a practice version of a visual search task that was to be performed during event-related scanning runs. (Behavioral and imaging data for the event-related scanning will be reported separately.) The screening tests were the Mini-Mental State Examination 
(Folstein et al., 1975); Beck Depression Inventory (Beck, 1978); vocabulary subtest of the Wechsler Adult Intelligence Scale-III (WAIS-III; Wechsler, 1997); corrected visual acuity (Bach, 1996), and the Dvorine color vision test (Dvorine, 1963). During this testing session before MRI scanning, participants also performed 9 tests of fluid cognition (perceptual speed, executive function, and memory), described in the later section, Cognitive Measures. Exclusion criteria were any one of the following: raw score less than 27 on Mini-Mental State Examination; score greater than 10 on the Beck Depression Inventory; scaled score less than the 50th percentile on WAIS-III vocabulary subtest; corrected visual acuity less than 20/40; score less than 12 on the Dvorine color vision test; or accuracy less than $75 \%$ in the visual search task. A total of 82 individuals were excluded, due to either psychometric screening scores (23 individuals), vision (9 individuals), handedness ( 4 individuals), health history (18 individuals), falling asleep during the resting-state scan (5 individuals), MRI technical difficulties (12 individuals), visual search task accuracy ( 5 individuals), or withdrawal from the study (6 individuals). For the final sample of 145 participants, DTI data were lost for 1 participant due to technical problems, but all other data are complete for the remaining participants. Demographic characteristics of the final 145 participants are presented in Table 1.

\subsection{MRI data acquisition}

We conducted imaging on a 3 T GE MR750 whole-body $60 \mathrm{~cm}$ bore MRI scanner (GE Healthcare, Waukesha, WI, USA) equipped with $50 \mathrm{mT} / \mathrm{m}$ gradients and a $200 \mathrm{~T} / \mathrm{m} / \mathrm{s}$ slew rate. An 8-channel head coil was used for radio frequency reception. Head motion was minimized with foam pads, and participants wore earplugs to reduce scanner noise. Imaging began with 3-plane (straight axial/ coronal/sagittal) localizer fast spin echo images that defined a volume for data collection. A semi-automated high-order shimming program ensured global field homogeneity. Data acquisition proceeded with 2 resting-state runs of $\mathrm{T} 2{ }^{*}$-weighted (functional) imaging sensitive to the blood oxygen-level-dependent signal, 4 or 5 runs of event-related, $12 *$-weighted imaging (to be reported in separate articles), 1 run of T1-weighted anatomical images, 2 runs of diffusion-weighted imaging (DWI), and 1 run of T2-weighted fluid-attenuated inversion recovery (FLAIR) imaging.

The T1-weighted anatomical images were 166 straight axial slices acquired with a 3D fast inverse-recovery-prepared spoiled gradient recalled (SPGR) sequence with $\mathrm{TR}=8.13 \mathrm{~ms}$, echo time $(\mathrm{TE})=3.18 \mathrm{~ms}$, inversion recovery time $(\mathrm{TI})=450 \mathrm{~ms}$, field of view

Table 1

Participant characteristics

\begin{tabular}{lllcl}
\hline Variable & M & SD & Age $r$ & Age $r^{2}$ \\
\hline Education (y) & 16.614 & 1.966 & $0.374^{* * *}$ & 0.140 \\
MMSE & 29.110 & 0.987 & $-0.241^{* *}$ & 0.058 \\
BDI & 2.517 & 2.713 & 0.162 & 0.026 \\
Vocabulary & 56.097 & 6.297 & 0.148 & 0.022 \\
Color vision & 13.883 & 0.400 & $-0.190^{*}$ & 0.036 \\
Visual acuity & -0.065 & 0.118 & $0.299^{* * *}$ & 0.089 \\
\hline
\end{tabular}

Note. $n=145$.

${ }_{* *}^{*}<0.05$ (uncorrected).

${ }^{* *} p<0.01$ (uncorrected).

${ }^{* * *} p<0.001$ (uncorrected).

Log MAR of 0 corresponds to Snellen 20/20, with negative values corresponding to better resolution. Thus, the positive correlation for acuity represents age-related decline in this measure.

Key: age $r$, correlation with age; age $r^{2}$, variance accounted for by age; BDI, score on Beck Depression Inventory (Beck, 1978); color vision, score on Dvorine color plates (Dvorine, 1963); M, mean; MMSE, raw score on Mini-Mental State Exam (Folstein et al., 1975); SD, standard deviation; visual acuity, logarithm of the minimum angle of resolution (MAR), for the Freiburg Visual Acuity Test (FRACT; Bach, 1996); vocabulary, raw score on the Wechsler Adult Intelligence Scale III (Wechsler, 1997).
$($ FOV $)=256 \mathrm{~mm} \times 256 \mathrm{~mm}$, flip angle $=12^{\circ}$, voxel size $=1 \times 1 \times$ $1 \mathrm{~mm}, 256 \times 256$ acquisition matrix, and a sensitivity encoding (SENSE) factor of 2 , using the array spatial sensitivity encoding technique and extended dynamic range.

The resting-state, $\mathrm{T} 2 *$-weighted gradient-echo EPI functional images were 29 contiguous slices acquired at an axial oblique orientation, parallel to the plane including the anterior and posterior commissures (AC-PC plane); $\mathrm{TR}=1500 \mathrm{~ms}, \mathrm{TE}=27 \mathrm{~ms}$, FOV $=$ $240 \mathrm{~mm} \times 240 \mathrm{~mm}$, flip angle $=77^{\circ}$, voxel size $=3.75 \times 3.75 \times$ $4 \mathrm{~mm}, 64 \times 64$ acquisition matrix, and a SENSE factor of 1 . Each of the 2 runs comprised a time series of 162 brain volumes. Four initial radio frequency excitations were performed to achieve steady state equilibrium and were subsequently discarded. Participants were instructed to remain awake with eyes open and to view a fixation cross throughout the run.

DWI data of 68 contiguous slices (parallel to the AC-PC plane) were acquired with a dual-echo spin-echo parallel EPI pulse sequence with the following parameters: $\mathrm{TR}=\min 9000 \mathrm{~ms}, \mathrm{TE}=$ $\min 81.3 \mathrm{~ms}, \mathrm{FOV}=256 \mathrm{~mm} \times 256 \mathrm{~mm}$, flip angle $=90^{\circ}$, voxel size $=1 \times 1 \times 2 \mathrm{~mm}$, acquisition matrix size $=128 \times 128$, and SENSE acceleration factor $=2$. The DWI scan used 30 diffusion-encoding directions (b-value $=1000 \mathrm{~s} / \mathrm{mm}^{2}$ ) with 1 nondiffusion-weighted $\mathrm{b}_{0}\left(0 \mathrm{~s} / \mathrm{mm}^{2}\right)$. Technical difficulty with DWI image acquisition led to the removal of DTI data for 1 older adult.

The T2-weighted FLAIR images were 39 contiguous slices with $\mathrm{TR}=\min 8000 \mathrm{~ms}, \mathrm{TE}=\min 120.156 \mathrm{~ms}, \mathrm{FOV}=240 \mathrm{~mm} \times 240 \mathrm{~mm}$, flip angle $=90^{\circ}$, voxel size $=0.9375 \times 0.9375 \times 3 \mathrm{~mm}, 256 \times 256$ acquisition matrix, and a SENSE factor of 1 . Slice orientation was axial oblique, parallel to the AC-PC plane.

\subsection{Cognitive measures}

We measured fluid cognitive performance from a battery of 9 tests, modified for computer administration (E-Prime; Psychology Software Tools, Sharpsburg, PA, USA) and selected to sample 3 domains of cognition: elementary perceptual speed, executive function, and memory, with 3 indicator variables per domain. Following the approach of Hedden et al. (2012, 2016), we selected the 3 tests for each domain on an a priori basis, and as described in a subsequent section (analyses) obtained a summary measure of each cognitive domain from a factor analysis of the 3 indicator variables.

\subsubsection{Speed}

The variables for the speed tests were mean RT for correct responses in: (a) simple RT; (b) choice RT; and (c) RT on neutral trials from a version of the Stroop task (Stroop, 1935). Simple and choice RT were each a block of 80 trials in which the participant either pressed a single response key at the onset of a white square (simple RT) or pressed a left or right response key depending on the direction of a left- or right-pointing arrow (choice RT). The Stroop task (described in the following paragraph) included compatible, incompatible, and neutral trials, and mean RT from the neutral trials was included in the perceptual speed domain. Individual trials were separated by a temporally jittered, blank interval, and RT was measured from display onset.

\subsubsection{Executive function}

The executive function variables included: (a) digit-symbol coding; (b) verbal fluency; and (c) Stroop interference. In the digit-symbol task (Salthouse, 1992), across 72 trials, a reference row of 9 pairs of digits and symbols remained displayed at the top of the screen, and participants made a yes/no key press response indicating whether a centrally presented probe digit-symbol pair corresponded to one of the reference pairs. The probe and reference pairs matched on $50 \%$ of the trials, and the digit symbol measure 
was mean RT for correct responses. The verbal fluency measures included both phonemic fluency, using the letters F, A, and S (Loonstra et al., 2001), and semantic fluency, using the animals category (Goodglass and Kaplan, 1972). Both measures were the number of items retrieved within 1 minute, which were then summed. The Stroop interference measure was a computeradministered version of the Stroop task (Stroop, 1935) in which 1 word was displayed in either red or blue on each trial and participants made a 2-choice manual response regarding the color of the word. The displayed word was either "red," "blue," "art," or "game" (the latter 2 neutral words were comparable in number of letters and word frequency to the color words). Across 120 trials, the word and displayed color were either compatible or incompatible or neutral (33\% of trials per condition). The interference score was the proportional increase in correct mean RT for responses in the incompatible condition relative to the compatible condition: (incompatible RT-compatible RT)/compatible RT.

\subsubsection{Memory}

The memory variables included: (a) the WAIS Digit Span subtest (Wechsler, 1997); (b) the delayed memory subtest from the California Verbal Learning Test (CVLT; Delis et al., 1987); and (c) a test of visual working memory similar to that reported by Saults and Cowan (2007). The digit-span measure was the mean of forward and backward spans. The CVLT measure involved presentation of 16 words for 3 seconds each, followed by immediate recall, and delayed recall following 20 minutes filled with the other testing procedures. The recall score was the number of words correctly recalled in the delayed test. The visual working memory test presented 2 displays of 6 squares, in unique and easily discriminable colors, for $1050 \mathrm{~ms}$ each, separated by a $1400 \mathrm{~ms}$ blank interval. The second display was either identical to the first or contained a color change in 1 of the 6 squares. At the presentation of the second display, participants made a 2-choice, key-press response as to whether or not they detected a color change. Participants completed 48 trials divided equally between color-change and no-change trials. The outcome variable was the percentage hits minus percentage false alarms.

To identify potential outliers, we examined regression models for each of the 9 tests, with age as a predictor. Three of the tests included 1 participant with a studentized residual $>3.5$, and these 3 data points were deleted before further analyses. Outcome measures were multiplied by -1 when necessary, so that positive values represented better performance for all tests.

\subsection{Imaging measures}

The imaging modalities comprised cerebral white-matter integrity, resting-state functional connectivity, and white-matter hyperintensity volume (Fig. 1). As was the case with the cognitive measures, within each imaging modality, we defined regionally specific summary measures from several indicator variables, in this case defined a priori from structural and functional anatomy. Across the 3 imaging modalities, there were 7 regionally specific measures. Within white-matter integrity, the 2 regionally specific measures were fractional anisotropy (FA) for sensorimotor and frontoparietal tracts. Within resting-state functional connectivity, we obtained 3 regionally specific measures, representing default mode, sensorimotor, and frontoparietal network connectivity. Within WMH volume, we distinguished between $2 \mathrm{WMH}$ categories, PV, and DWM. Additional details regarding the imaging methods are provided in Supplementary Material.

\subsubsection{Cerebral white-matter integrity}

From DTI tractography on the DWI data (Fig. 1, panel A), we distinguished 2 types of white-matter tracts, those connecting primarily sensorimotor regions, and those connecting primarily frontoparietal association cortex. The indicator variable, for each tract, was the average FA, which represents the relative directionality of the diffusion of molecular water (Basser and Pierpaoli, 1996; Beaulieu, 2014). Because tracts with highly aligned axons will exhibit a high degree of directionality of diffusion, FA is a useful though indirect measure of the microstructural organization of white-matter (Jones et al., 2013). The sensorimotor tracts included the corticospinal tract, optic radiations (posterior thalamic radiations), and inferior longitudinal fasciculus. The frontoparietal tracts included the genu and splenium of the corpus callosum, and the superior longitudinal fasciculus. The corticospinal tract, optic radiations, and superior and inferior longitudinal fasciculi were defined within each cerebral hemisphere separately, and the genu and splenium were divided at the midline to yield left and right hemisphere values. Thus, the sensorimotor and frontoparietal tracts each comprised 6 FA values as indicator variables.

To define individual white-matter tracts, we performed deterministic tractography of the DTI data, in each participant's native space. Following visual inspection of the DWI images for sufficient quality, we concatenated the $2 \mathrm{DWI}$ runs into a $4 \mathrm{D}$ volume and preprocessed the DWI images with DTIPrep (Liu et al., 2010), including slice-wise, interlace-wise, and gradient-wise intensity artifact correction, as well as eddy-current distortion and motion correction. To match these applied corrections, we reoriented the diffusion directions. We performed tensor fitting using DSI Studio (Yeh et al., 2013; http://dsi-studio.labsolver.org/) to generate a DTI image and subsequently extracted an FA map in each participant's native space. Each tensor image was visually inspected for quality before creation of the FA map.

For each tract, we selected a single seed region from the parcellation map of the ICBM DTI-81 white-matter atlas (Mori et al., 2008, but cf.; Rohlfing, 2013), which was deprojected into native space. Fiber tracking was performed on the diffusion images for each participant using DSI Studio, which defined white-matter fibers coursing through the seed region, in conjunction with inclusion and exclusion imaging planes that constrained the location of the fibers.

Following an approach similar to that of Sasson et al. (2013), the tractography methods incorporated information from both standard atlas and study-specific data. This approach has the advantage of defining tracts within each participant's native space, while providing a common metric across participants by using standardized anatomical and study-specific criteria to define tracts. Once streamlines were acquired via tractography they were exported in voxel space (without interpolation) following the tract density imaging method of Calamante et al. (2010). We then created a study-specific mask for each tract that represented voxels common to $75 \%$ of the first 120 participants (40 each within younger, middle-aged, and older age groups) in the standard space of the ICBM template. Within native space, we then masked each participant's tracts with the binarized study-specific tracts to constrain any extraneous streamlines. For each participant, following diffusion image reconstruction, we obtained FA values at each voxel and averaged the values within each tract.

\subsubsection{Resting-state functional connectivity}

From the fMRI resting-state functional connectivity data (Fig. 1, Panel B), we identified individual networks from an independent component analysis (ICA). We focused on 3 types of networks relevant for fluid cognition: a default mode network comprising medial prefrontal and medial posterior cortical regions, networks for sensorimotor cortical regions, and networks for frontoparietal cortical regions. 
A

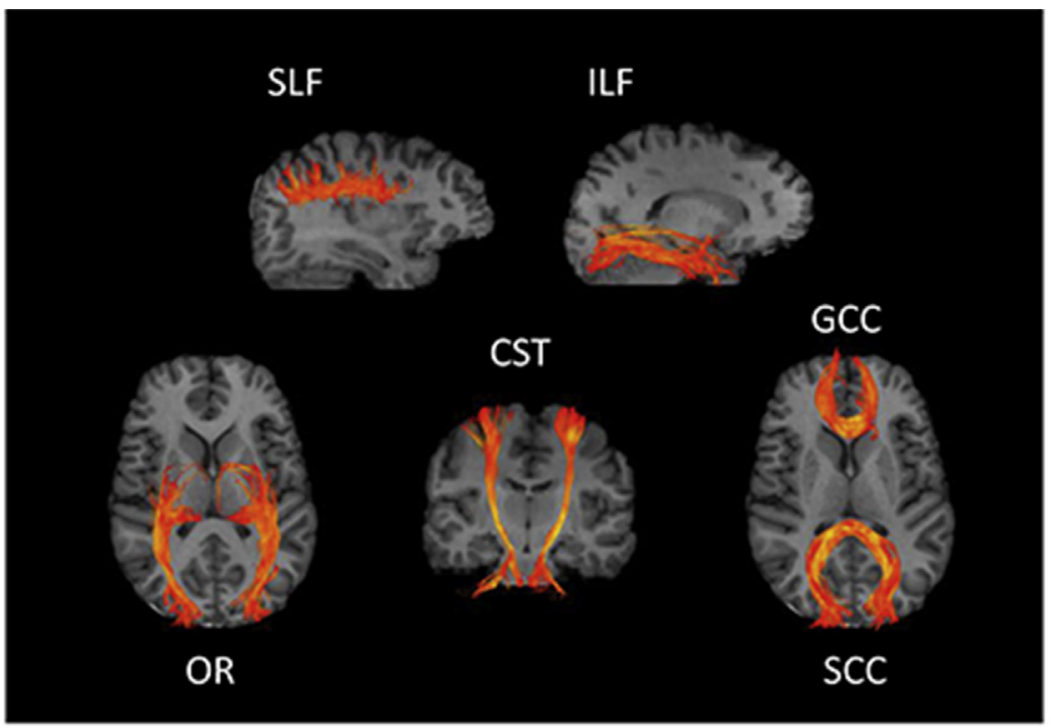

B

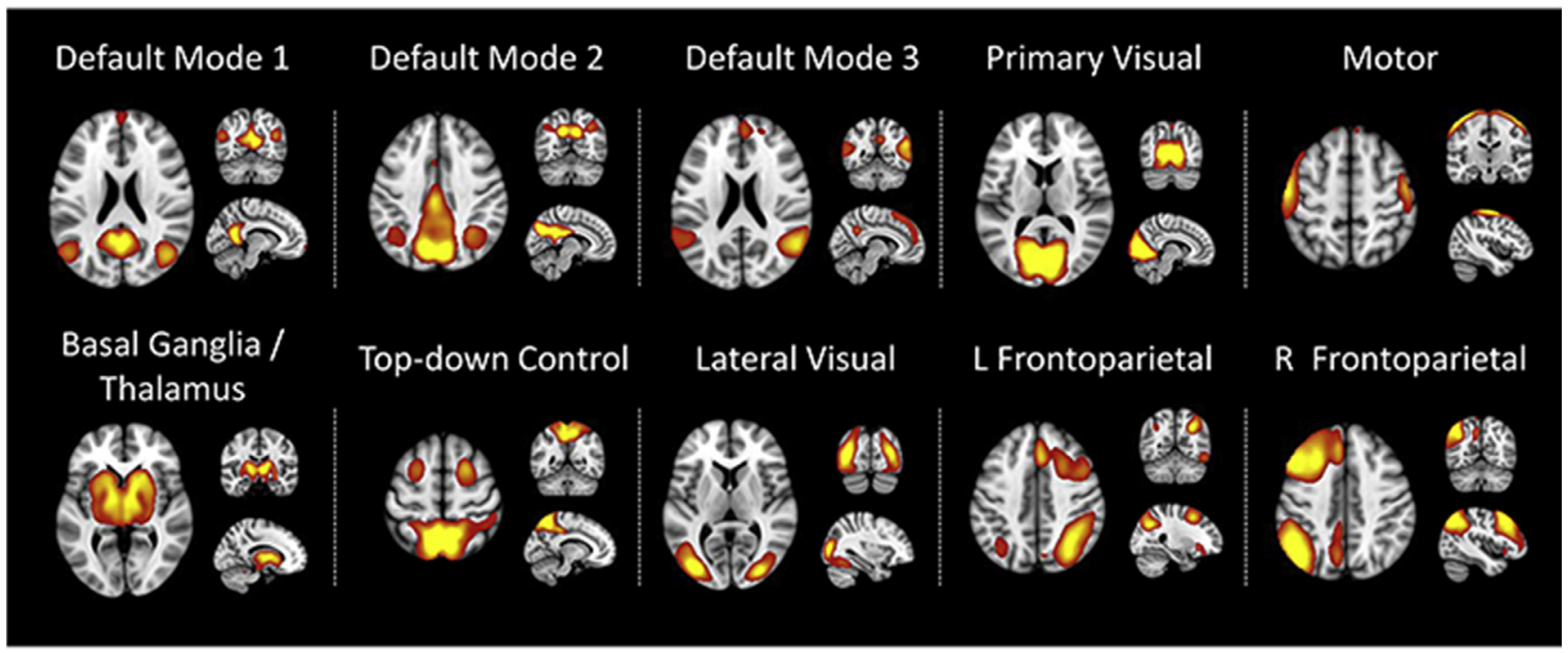

C

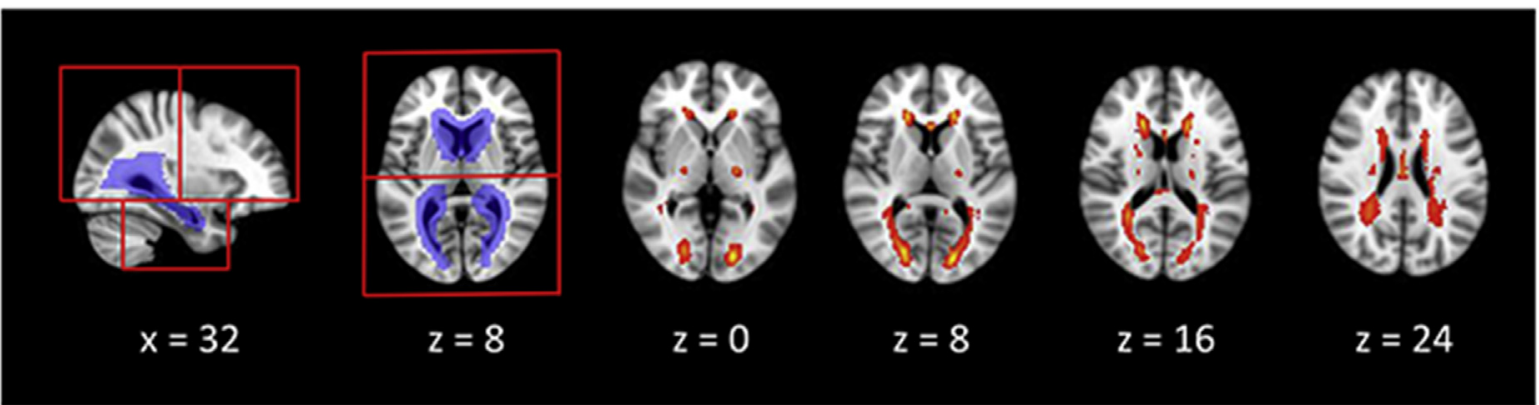

Fig. 1. Data from different brain imaging modalities. (A), fractional anisotropy (FA) of white-matter tracts derived from deterministic tractography of diffusion tensor imaging (DTI) data; examples are tracts for a representative participant. (B), 10 components of resting-state functional connectivity, for all participants combined, obtained from independent component analysis (ICA); note that data are presented in radiological orientation (left = right); ICA component labels correspond to the Laird et al. (2011) templates. (C), map of white-matter hyperintensities (WMHs), for all participants combined, overlaid on the MNI template brain. In the 2 left-most images, the periventricular (PV) mask is depicted in blue. The red borders represent the boundaries of 3 regions from which WMHs were estimated. Hyperintensities were classified as being either PV (within the mask) or deep white matter (DWM; outside the mask). Hyperintensity volumes were obtained for the PV and DWM categories within each of the 3 anatomical regions, yielding 6 WMH volume variables for each participant. Abbreviations: CST, corticospinal tract; GCC, genu of corpus callosum; ILF, inferior longitudinal fasciculus; OR, optic radiations; SCC, splenium of corpus callosum; SLF, superior longitudinal fasciculus. (For interpretation of the references to color in this figure legend, the reader is referred to the Web version of this article.) 
Preprocessing of the resting-state fMRI data began with an inhouse pipeline comprising custom Python code and several tools in FSL 5.0.5 (Smith et al., 2004; http://www.fmrib.ox.ac.uk/fsl): slicetimer for slice-time correction, MCFLIRT for motion correction, BET for brain extraction, and FLIRT for normalization to the MNI152 T1 template (Montreal Neurological Institute, Montreal, Canada). We regressed out signal from white-matter and cerebrospinal fluid on the basis of masks created in FSL FAST (Zhang et al., 2001) and smoothed the data with a 5-mm kernel using FSL SUSAN (Smith and Brady, 1997). Temporal band-pass filtering limited the data to frequencies in the $0.001-0.08 \mathrm{~Hz}$ band. Following the recommendation of Power et al. (2012), we performed motion scrubbing using a framewise displacement threshold of 0.5 and timecourse variance threshold of $0.5 \%$. Motion scrubbing led to the removal of 1 timepoint from 1 participant. Global signal regression was not performed, because the global negative index (Chen et al., 2012) was greater than the critical value of 0.03 for each participant, suggesting that global signal regression would not be beneficial.

We entered each participant's preprocessed data into an ICA using FSL MELODIC (Beckmann et al., 2005) with the number of components fixed at 20. Resulting components were identified using linear template-matching (Greicius et al., 2007), selecting components that best fit a priori defined network templates (Laird et al., 2011). Following the method of Greicius et al., we obtained goodness-of-fit scores by taking the average $z$ score of voxels within the template minus the average $z$ score of voxels outside the template and selecting the component in which the difference was greatest. We selected 10 relevant components (Fig. 1, panel B), with a goodness-of-fit score of at least 1.5. To create masks for each of these 10 components, we thresholded the component's corresponding template network at $\mathrm{z}=4$ and then binarized the thresholded template network.

We entered the resting-state data into a dual regression using FSL. The ICA spatial maps were regressed into each participant's data set (spatial regression), resulting in matrices that reflect the temporal dynamics for each component and participant. These timecourses were regressed into each participant's data set (temporal regression) to estimate participant-specific spatial maps, standardized into $z$ score maps, and masked by the corresponding Laird et al. (2011) template mask. This yielded 10 outcome measures, from the mean of the absolute values of all non-zero voxels within each mask, as a measure of functional connectivity per component and participant. As illustrated in Fig. 1 (panel B), our estimate of the default mode network was based on 3 ICA components, with slightly different shapes, which matched the Laird et al. (2011) default mode network template. The sensorimotor networks comprised 3 ICA components matching the Laird et al. templates for primary visual, motor, and basal ganglia/thalamus networks. The frontoparietal networks comprised 4 ICA components matching the Laird et al. templates for top-down control, lateral visual, left frontoparietal, and right frontoparietal networks.

\subsubsection{WMH volume}

We calculated WMH volume with the Lesion Segmentation Tool within SPM8 (Schmidt et al., 2012; http://www.applied-statistics. de/lst.html), distinguishing WMHs located near the lateral ventricles (periventricular; PV) from those located in DWM. WMH estimation was conducted on the T2-weighted FLAIR images (coregistered to the T1-weighted image), at the participant level, in native space (Fig. 1, panel C). Initially, voxels in the T1-weighted image were segmented into 3 tissue classes of cerebrospinal fluid, gray matter, and white matter. Hyperintense outliers in the T2-weighted FLAIR images (in native T1-space) were seeded within the gray and white matter, and a growth algorithm created a lesion probability map for each participant. Lesion probability maps were then masked with the ALVIN (Automatic Lateral Ventricle delineation) mask of the lateral ventricles (Kempton et al., 2011), which we extended to include the white matter adjacent to the posterior horns of the lateral ventricles. An in-house Python script classified hyperintensities located within the mask as PV, and those located outside of the mask as DWM. The intensity distribution threshold was set higher for PV (kappa $=0.50$ ) than for DWM (kappa $=0.30$ ) to reduce the likelihood of false identification of WMHs in the watershed area of the PV white matter.

To further categorize WMH location, each participant's lesion map was subdivided into 3 regions: 2 superior regions ( 1 anterior and 1 posterior) and 1 inferior region (Fig. 1, panel C). These regions were defined by planes placed on a group-averaged T2-weighted structural template from the first 120 participants (40 per younger, middle-aged, and older adults), which were then deprojected to participant-specific native space. Each of the 3 regions contained labeled PV and DWM hyperintensities, yielding $6 \mathrm{WMH}$ values for each participant. The WMH volumes were defined as percentages of intracranial volume to control for individual differences in brain size. Because WMH volume tends to increase logarithmically with adult age (Raz et al., 2012), the data for each of the $6 \mathrm{WMH}$ variables were log-transformed for further analyses.

\subsection{Analyses}

As described in the previous section, the behavioral measures included 3 domains of fluid cognition: elementary perceptual speed, executive function, and memory, with 3 indicator variables per domain. We used the first unrotated factor from a factor analysis of all 9 cognitive tests, partialled for WAIS vocabulary and gender, as a general summary measure for fluid cognition. To define the domain-specific summary measures, we used an approach similar to that of Hedden et al. (2012, 2016). We extracted the first unrotated factor from a factor analysis of the 3 tests in each domain. We conducted a principal axis factor analysis on the relevant indicator variables (for all participants) and used the factor score (for each participant) from the first unrotated factor as the summary measure. For both the general and domain-specific analyses, we used a principal factor analysis rather than a principal components analysis because our interest was in the shared variance among the indicator variables rather than in the mathematically independent components of the total variance. The factor scores served as summary measures for the purposes of data reduction. We do not claim that the behavioral (or imaging) data have a particular factor structure.

To adjust the domain-specific summary measures for the variance shared with related indicator variables, we created a residual score that partialled all of the other indicator variables, similar to Salthouse et al. (2015) and Karama et al. (2011). ${ }^{1}$ For example, a residual speed measure was created by partialling the speed factor (based on 3 indicator variables) for the other 6 cognitive measures contributing to executive function and memory. All residual factor

\footnotetext{
1 Our approach to controlling for shared variance is somewhat different from that of Salthouse et al. (2015) and Karama et al. (2011), in that these authors partialled out the first factor from the variable of interest. However, because the variable of interest is also contained in the first factor, it is possible that this approach is too conservative. We therefore partial out other individual variables not associated with the variable of interest. For example, in creating the residual speed measure (from the factor score for the 3 speed variables), we partialled out the other 9 cognitive indicator variables not associated with the speed factor. Another possible approach would be to partial out the executive function and memory factor scores from the speed factor score. While conceptually similar to our approach, we believe that partialling the factor scores may not be as sensitive to potential shared variance with the individual indicator variables.
} 
scores were also partialled for WAIS vocabulary (as an estimate of verbal IQ) and gender.

We used a similar method to define regionally specific and general summary measures within the 3 imaging modalities of cerebral white-matter integrity, resting-state functional connectivity, and WMH volume. We created a general summary measure from the first unrotated factor for all of the indicator variables: white-matter integrity (12 tract FA variables), resting-state functional connectivity (10 ICA component variables), and $\mathrm{WMH}$ (six regional variables), partialled for WAIS vocabulary and gender. The indicator variables for white-matter integrity were grouped into sensorimotor and frontoparietal tracts; those for resting-state functional connectivity were grouped into default mode, sensorimotor, and frontoparietal networks; and those for WMH volume were grouped into PV and DWM categories. As with the cognitive measures, we created a residual, domain-specific measure by partialling all domain-associated variables not associated with the regionally specific variables. In the case of white-matter integrity, for example, we conducted separate factor analyses of the FA variables for the sensorimotor and frontoparietal tracts and used the first unrotated factor, from each analysis, as the regionally specific summary measure. We then created residual measures by partialling the remaining indicator variables within each imaging modality. For example, a residual sensorimotor FA measure was created by partialling the sensorimotor FA factor score (based on 6 tract FA indicator variables) for the other 6 FA variables associated with the frontoparietal tracts.

Factor loadings of indicator variables on the general and specific factors are presented in Table 2. Because some of the indicator variables were highly correlated, variance estimates were biased and exceeded 1.0 in some instances. The table therefore lists the squared multiple correlations among the indicator variables for the domain- and regionally specific-factors, rather than variance estimates. The general factor squared multiple correlation values were 0.854 for fluid cognition, 0.972 for white-matter integrity, 0.742 for resting-state functional connectivity, and 0.854 for WMH volume.

Correlation matrices of the general and domain-specific cognitive and imaging measures, with age included and age partialled, are presented in Supplementary Material (Table S1).

Analyses were conducted using ordinary least squares regression (SAS 9.4, SAS Institute, Inc., Cary, NC, USA) and conditional process (mediation) analyses as implemented in the PROCESS macro for SAS (Hayes, 2013). In the mediation analyses, parameter estimates and bootstrap confidence intervals were based on 10,000 percentile-based bootstrap samples. Significant indirect effects are defined by a confidence interval not including zero. In view of the large number of statistical tests conducted, we adopted conservative significance levels of 0.01 and 0.001 for parametric tests and set bootstrap confidence intervals to $99 \%$.

\section{Results}

\subsection{General fluid cognition and imaging modalities}

\subsubsection{Age correlations}

The general summary measures for fluid cognition, whitematter integrity (FA), and resting-state connectivity exhibited significant age-related decline, whereas the general measure of WMH volume increased with age (Fig. 2). The age-related decline in general fluid cognition remained significant when partialled for the general measures of white-matter integrity, resting-state functional connectivity, and WMH volume, $r=-0.517, p<0.001$. Similarly, the general imaging measures continued to exhibit age-related decline when partialled for fluid cognition: $r=-0.498, p<0.001$, for whitematter integrity, $r=-0.386, p<0.001$, for resting-state functional
Table 2

Factor loadings

\begin{tabular}{|c|c|c|}
\hline \multirow[t]{2}{*}{ Variable } & \multicolumn{2}{|c|}{ Factor loading } \\
\hline & Specific & General \\
\hline \multicolumn{3}{|l|}{ Fluid cognition } \\
\hline \multicolumn{3}{|l|}{ Speed (factor SMC $=0.706$ ) } \\
\hline Simple RT & 0.612 & 0.600 \\
\hline Choice RT & 0.743 & 0.703 \\
\hline Stroop neutral RT & 0.721 & 0.795 \\
\hline \multicolumn{3}{|l|}{ Executive function (factor SMC $=0.547$ ) } \\
\hline Verbal fluency & 0.546 & 0.525 \\
\hline Stroop interference RT & 0.332 & 0.486 \\
\hline Digit symbol & 0.679 & 0.772 \\
\hline \multicolumn{3}{|l|}{ Memory (factor SMC $=0.333$ ) } \\
\hline Digit span & 0.383 & 0.379 \\
\hline Delayed recall & 0.432 & 0.431 \\
\hline Visual working memory & 0.363 & 0.483 \\
\hline \multicolumn{3}{|l|}{ White-matter integrity } \\
\hline \multicolumn{3}{|l|}{ Sensorimotor FA (factor SMC $=0.929$ ) } \\
\hline Left CST & 0.692 & 0.515 \\
\hline Right CST & 0.678 & 0.550 \\
\hline Left optic radiations & 0.842 & 0.806 \\
\hline Right optic radiations & 0.827 & 0.758 \\
\hline Left inferior longitudinal fasciculus & 0.770 & 0.797 \\
\hline Right inferior longitudinal fasciculus & 0.786 & 0.747 \\
\hline \multicolumn{3}{|l|}{ Frontoparietal FA (factor SMC $=0.961$ ) } \\
\hline Left genu & 0.925 & 0.871 \\
\hline Right genu & 0.913 & 0.864 \\
\hline Left splenium & 0.863 & 0.797 \\
\hline Right splenium & 0.888 & 0.843 \\
\hline Left superior longitudinal fasciculus & 0.787 & 0.764 \\
\hline Right superior longitudinal fasciculus & 0.689 & 0.689 \\
\hline \multicolumn{3}{|l|}{ Resting-state FC } \\
\hline \multicolumn{3}{|l|}{ Default mode FC (factor SMC $=0.429$ ) } \\
\hline Default mode 1 & 0.437 & 0.471 \\
\hline Default mode 2 & 0.479 & 0.635 \\
\hline Default mode 3 & 0.491 & 0.513 \\
\hline \multicolumn{3}{|c|}{ Sensorimotor resting-state FC (factor SMC $=0.237$ ) } \\
\hline Primary visual & 0.345 & 0.463 \\
\hline Motor cortex & -0.163 & 0.036 \\
\hline Basal ganglia/thalamus & 0.388 & 0.417 \\
\hline \multicolumn{3}{|c|}{ Frontoparietal resting-state FC (factor SMC $=0.433$ ) } \\
\hline Top-down control & 0.394 & 0.415 \\
\hline Lateral visual & 0.359 & 0.370 \\
\hline Left frontoparietal & 0.347 & 0.353 \\
\hline Right frontoparietal & 0.524 & 0.592 \\
\hline \multicolumn{3}{|l|}{ White-matter hyperintensity (WMH) volume } \\
\hline \multicolumn{3}{|l|}{ Periventricular (factor SMC $=0.773$ ) } \\
\hline Superior anterior & 0.791 & 0.747 \\
\hline Superior posterior & 0.819 & 0.837 \\
\hline Inferior & 0.582 & 0.600 \\
\hline \multicolumn{3}{|l|}{ Deep white matter $($ factor SMC $=0.621)$} \\
\hline Superior anterior & 0.683 & 0.630 \\
\hline Superior posterior & 0.577 & 0.640 \\
\hline Inferior & 0.603 & 0.588 \\
\hline
\end{tabular}

Note. SMC, squared multiple correlations of indicator variables with the associated factor.

Specific factor loadings are the loadings of the indicator variables on the associated domain-specific factor; general factor loadings are the loadings of the indicator variables on the general factor of all the indicator variables within the associated categories of fluid cognition, white-matter integrity, resting-state functional connectivity, and WMH volume.

Key: CST, corticospinal tract; FA, fractional anisotropy; FC, functional connectivity; $\mathrm{RT}$, reaction time.

connectivity, and $r=0.380, p<0.001$, for WMH volume. Thus, age exhibited strong, independent relations to the summary measures of fluid cognition and the imaging modalities. Additional regression models with quadratic $\left(a^{2} e^{2}\right)$ predictor variables did not yield significant nonlinear effects.

\subsubsection{Tests of mediation and moderation}

To determine whether the general imaging measures were mediators of the relation between age and general fluid cognition, 
we conducted mediation analyses of a model in which age was a predictor $(x)$ of fluid cognition $(y)$, and the general summary measures (factor scores) for white-matter integrity, resting-state functional connectivity, and WMH volume were mediators $(m)$ of the relation between age and fluid cognition. We tested both the general and domain-specific measures of cognition as outcome variables. This is essentially a path model in which the relation of age to each of the mediators is a separate $a$ path, the relation of each of the mediators to fluid cognition (controlling for age) is a separate $b$ path, the relation of age to fluid cognition (the total effect of age) is the $c$ path, and the direct effect of age (controlling for all of the mediators) is the $c^{\prime}$ path. The mediators were modeled as operating in parallel, and thus each mediator was covaried for the others. A significant $a \times b$ path interaction for a mediator variable would imply that the effect of the predictor (age) on the outcome variable (fluid cognition) is indirect, operating through that variable, rather than direct. The $a \times b$ path interaction effects were tested with bootstrap confidence intervals, as recommended by Hayes (2013).

Results for the general imaging measures are presented in Table 3. The effects of age on all of the mediators ( $a$ paths) were significant, as was the total effect of age ( $c$ path) on fluid cognition and residual executive function, corresponding to the pattern of the correlations noted previously. No mediating effects, however, were significant. None of the general imaging variables exhibited either a significant influence on fluid cognition controlling for age ( $b$ path) or a significant $a \times b$ interaction. For residual executive function, the general imaging variables combined led to a decrease in the direct effect of age, relative to the total effect, but none of the general imaging variables was significant as a mediator.

A

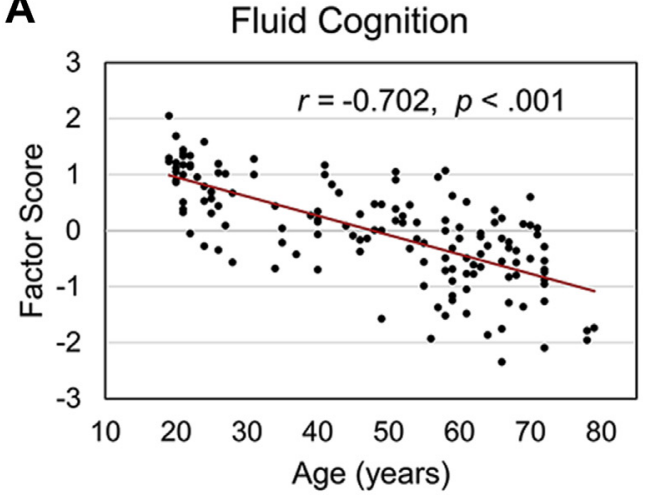

C

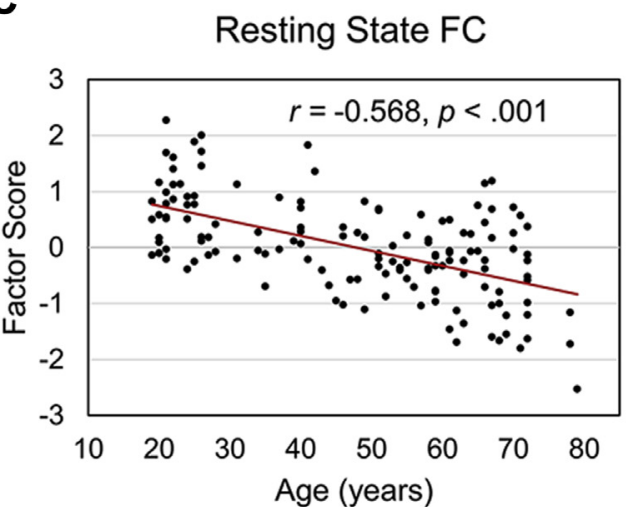

We also conducted tests of age moderation for each of the mediators, in terms of the age $\times$ mediator interaction in the prediction of fluid cognition, and none of these interactions was significant.

\subsection{Specific cognitive and imaging variables}

\subsubsection{Age correlations}

The age correlations of the domain-specific cognitive and regionally specific imaging variables are presented in Table 4. Before partialling the shared variance among the indicator variables, the 3 cognitive domains each exhibited significant agerelated decline, as did the 7 regionally specific measures within the modalities of white-matter integrity, resting-state functional connectivity, and WMH volume. The residual scores represent the 3 cognitive domains and 7 regionally specific imaging measures partialled for the variance shared with the other indicator variables (see the previous section, analyses, in materials and methods). Of the residual cognitive measures, only executive function continued to exhibit significant age-related decline. Of the residual imaging measures, we observed significant age-related decline in frontoparietal FA, sensorimotor resting-state functional connectivity, and an age-related increase in PV WMH volume.

For the residual executive function measure, the independent age-effects model did not hold. The age-residual executive correlation was no longer significant when partialled for all of the regionally specific imaging measures (i.e., 2 white-matter integrity, 3 resting-state functional connectivity, and $2 \mathrm{WMH}$ volume measures), $r=-0.069, p>0.40$. In contrast, for the 3 residual imaging measures that exhibited age-related decline (Table 4), each continued to show a significant age-related effect when partialled
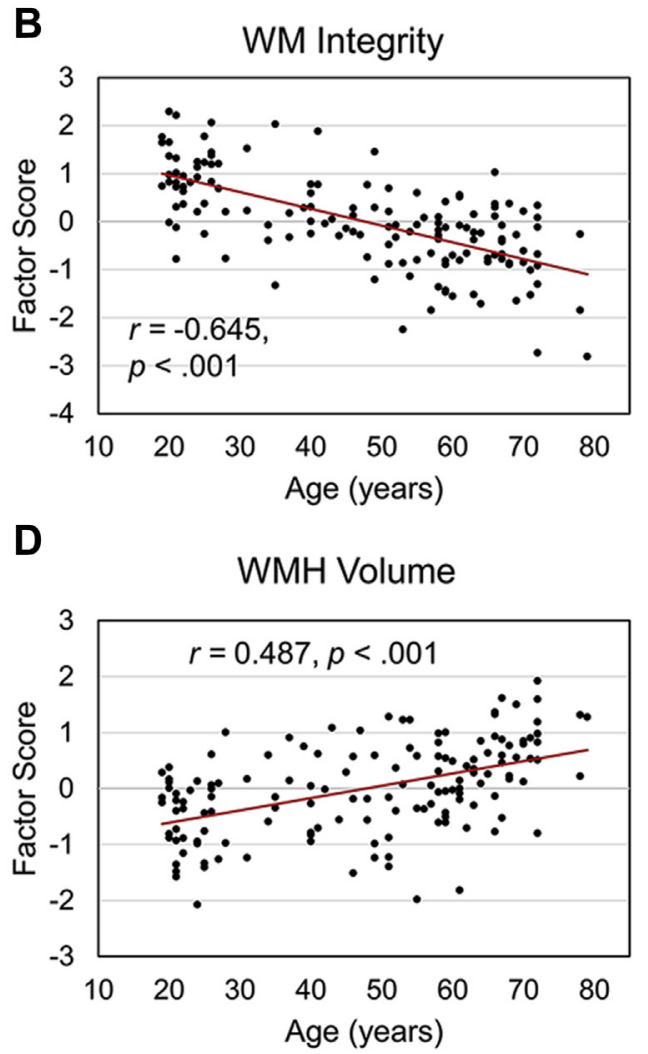

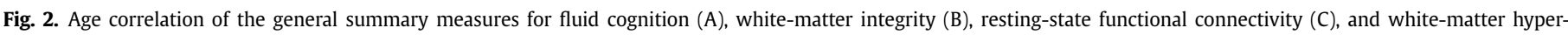
intensity volume (D). Abbreviations: FC, functional connectivity; WM, white matter; WMH, white-matter hyperintensity. 
Table 3

General imaging variables as mediators of age-fluid cognition relation

\begin{tabular}{|c|c|c|c|c|c|c|c|}
\hline Model & Effect & $S E$ & $\beta$ & $t$ & $p$ & Lower CI & Upper CI \\
\hline \multicolumn{8}{|l|}{$x \rightarrow m$ ( $a$ path; for all models) } \\
\hline Age $\rightarrow$ General WM integrity & -0.0351 & 0.0035 & -0.6409 & -10.0238 & 0.0001 & -0.0442 & -0.0259 \\
\hline Age $\rightarrow$ General RS FC & -0.0273 & 0.0033 & -0.5677 & -8.3521 & 0.0001 & -0.0359 & $-\mathbf{0 . 0 1 8 8}$ \\
\hline Age $\rightarrow$ General WMH volume & 0.0219 & 0.0034 & 0.4875 & 6.5020 & 0.0001 & 0.0131 & 0.0306 \\
\hline \multicolumn{8}{|c|}{$\begin{array}{l}\text { Model } 1 \text { (general fluid cognition): } x=\text { age; } m=\text { general WM integrity; general RS FC; general WMH volume; } y=\text { General fluid cognition } \\
\qquad m \rightarrow y \text { ( } b \text { path) }\end{array}$} \\
\hline General WM integrity $\rightarrow$ Gen Fluid Cogn & 0.0297 & 0.0741 & 0.0377 & 0.4012 & 0.6889 & -0.1639 & 0.2233 \\
\hline General RS FC $\rightarrow$ Gen Fluid Cogn & 0.1097 & 0.0788 & 0.1102 & 1.3923 & 0.1661 & -0.0962 & 0.3156 \\
\hline General WMH volume $\rightarrow$ Gen Fluid Cogn & 0.0363 & 0.0767 & 0.0346 & 0.4729 & 0.6370 & -0.1642 & 0.2368 \\
\hline \multicolumn{8}{|l|}{ Mediation effects ( $a \times b$ interaction) } \\
\hline General WM integrity & -0.0010 & 0.0027 & -0.0242 & - & - & -0.0085 & 0.0060 \\
\hline General RS FC & -0.0030 & 0.0021 & -0.0626 & - & - & -0.0083 & 0.0026 \\
\hline General WMH volume & 0.0008 & 0.0016 & 0.0169 & - & - & -0.0039 & 0.0049 \\
\hline Total effect for age (c path) & 0.0347 & 0.0030 & -0.6981 & -11.6006 & 0.0001 & -0.0425 & -0.0269 \\
\hline Direct effect for age (c' path) & -0.0314 & 0.0045 & -0.6282 & -7.0487 & 0.0001 & -0.0431 & -0.0198 \\
\hline \multicolumn{8}{|c|}{$\begin{array}{l}\text { Model } 2 \text { (residual speed): } x=\text { age; } m=\text { general WM integrity; general RS FC; general WMH volume; } y=\text { residual speed } \\
\quad m \rightarrow y \text { (b path) }\end{array}$} \\
\hline General WM integrity $\rightarrow$ residual speed & 0.0086 & 0.0663 & 0.0300 & 0.1296 & 0.8971 & -0.1646 & 0.1818 \\
\hline General RS FC $\rightarrow$ residual speed & 0.0435 & 0.0705 & 0.0726 & 0.6174 & 0.5380 & -0.1406 & 0.2276 \\
\hline General WMH volume $\rightarrow$ residual speed & 0.0227 & 0.0686 & 0.0342 & 0.3312 & 0.7410 & -0.1566 & 0.2021 \\
\hline \multicolumn{8}{|l|}{ Mediation effects ( $a \times b$ interaction) } \\
\hline General WM integrity & -0.0003 & 0.0024 & -0.0192 & - & - & -0.0066 & 0.0061 \\
\hline General RS FC & -0.0012 & 0.0021 & -0.0412 & - & - & -0.0068 & 0.0046 \\
\hline General WMH volume & 0.0005 & 0.0014 & 0.0167 & - & - & -0.0035 & 0.0043 \\
\hline Total effect for age ( $c$ path) & -0.0046 & 0.0027 & -0.1296 & -1.7430 & 0.0835 & -0.0116 & 0.0023 \\
\hline Direct effect for age ( $c^{\prime}$ path) & -0.0036 & 0.0040 & -0.0858 & -0.9115 & 0.3636 & -0.0141 & 0.0068 \\
\hline \multicolumn{8}{|c|}{$\begin{array}{l}\text { Model } 3 \text { (residual executive): } x=\text { age; } m=\text { general WM integrity; general RS FC; general WMH volume; } y=\text { residual executive } \\
\quad m \rightarrow y \text { (b path) }\end{array}$} \\
\hline General WM integrity $\rightarrow$ residual executive & -0.0424 & 0.0547 & -0.0872 & -0.7758 & 0.4392 & -0.1853 & 0.1005 \\
\hline General RS FC $\rightarrow$ residual executive & 0.0249 & 0.0582 & 0.0448 & 0.4278 & 0.6695 & -0.1271 & 0.1768 \\
\hline General WMH volume $\rightarrow$ residual executive & 0.0086 & 0.0566 & 0.0151 & 0.1525 & 0.8790 & -0.1393 & 0.1566 \\
\hline \multicolumn{8}{|l|}{ Mediation effects ( $a \times b$ interaction) } \\
\hline General WM integrity & 0.0015 & 0.0020 & 0.0559 & - & - & -0.0037 & 0.0071 \\
\hline General RS FC & -0.0007 & 0.0017 & -0.0254 & - & - & -0.0053 & 0.0039 \\
\hline General WMH volume & 0.0002 & 0.0013 & 0.0074 & - & - & -0.0030 & 0.0036 \\
\hline Total effect for age ( $c$ path) & -0.0061 & 0.0022 & -0.2333 & -2.7924 & 0.0060 & $-\mathbf{0 . 0 1 1 9}$ & -0.0004 \\
\hline Direct effect for age ( $c^{\prime}$ path) & -0.0071 & 0.0033 & -0.2712 & -2.1644 & 0.0322 & -0.0157 & 0.0015 \\
\hline \multicolumn{8}{|c|}{$\begin{array}{l}\text { Model } 4 \text { (residual memory): } x=\text { age; } m=\text { general WM integrity; general RS FC; general WMH volume; } y=\text { residual memory } \\
\quad m \rightarrow y \text { (b path) }\end{array}$} \\
\hline General WM integrity $\rightarrow$ residual memory & 0.0112 & 0.0524 & 0.0287 & 0.2128 & 0.8318 & -0.1257 & 0.1481 \\
\hline General RS FC $\rightarrow$ residual memory & 0.0035 & 0.0557 & 0.0100 & 0.0622 & 0.9505 & -0.1421 & 0.1490 \\
\hline General WMH volume $\rightarrow$ residual memory & -0.0148 & 0.0543 & -0.0260 & -0.2734 & 0.7850 & -0.1566 & 0.1269 \\
\hline \multicolumn{8}{|l|}{ Mediation effects ( $a \times b$ interaction) } \\
\hline General WM integrity & -0.0004 & 0.0020 & -0.0184 & - & - & -0.0058 & 0.0048 \\
\hline General RS FC & -0.0001 & 0.0013 & -0.0057 & - & - & -0.0033 & 0.0037 \\
\hline General WMH volume & -0.0003 & 0.0012 & -0.0127 & - & - & -0.0038 & 0.0026 \\
\hline Total effect for age ( $c$ path) & -0.0032 & 0.0021 & -0.1240 & -1.5210 & 0.1305 & -0.0087 & 0.0023 \\
\hline Direct effect for age ( $c^{\prime}$ path) & -0.0024 & 0.0032 & -0.0872 & -0.7545 & 0.4519 & -0.0106 & 0.0059 \\
\hline
\end{tabular}

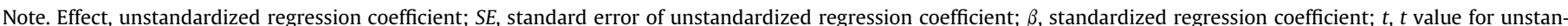

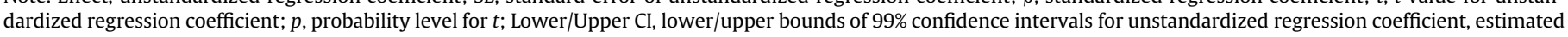
from bootstrap sampling with 10,000 samples.

Significant effects are presented in bold.

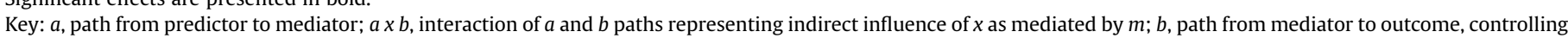

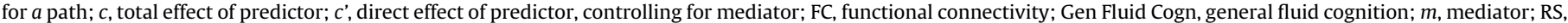
resting state; WM, white matter; $x$, predictor variable; $y$, outcome variable.

for the 3 residual cognitive measures: $r=-0.298, p<0.001$, for residual frontoparietal FA; $r=-0.342, p<0.001$, for residual sensorimotor resting-state functional connectivity, and $r=0.320, p$ $<0.001$, for PV WMH volume.

\subsubsection{Tests of mediation and moderation}

For each of the 3 residual cognitive measures of speed, executive function, and memory, we tested a mediation model in which the 7 regionally specific imaging measures ( 2 white-matter integrity, 3 resting-state functional connectivity, and $2 \mathrm{WMH}$ volume measures) were parallel mediators of the relation between age and the residual cognitive measure. In these 3 models, the mediators were the regionally specific imaging factor scores obtained before partialling (i.e., not residuals). Because the mediation analysis covaries each mediator for the others, shared variance is controlled in model estimation.
It may not seem intuitive to include, in this analysis, all of the imaging and cognitive variables, when they did not all exhibit significant age-related effects. However, researchers have recently argued that mediators should be selected on theoretical rather than empirical grounds, and that an indirect effect of a predictor may be valid and interpretable even in the absence of a significant total effect (Hayes, 2009, 2013; Mackinnon and Fairchild, 2009; Shrout and Bolger, 2002).

The mediation effects for the 3 models are presented in Table 5 . Note that the $a$ paths between age and the mediators are identical for the 3 models and are thus just listed once. No mediators of the relation of age to either residual speed or residual memory were significant. Resting-state functional connectivity of sensorimotor networks, however, was a significant mediator ( $a \times b$ path interaction) of age-related decline in residual executive function (Fig. 3 ). 
Table 4

Age correlations of specific cognitive and imaging factors

\begin{tabular}{|c|c|c|}
\hline Factor & $\begin{array}{l}r \text { with age before } \\
\text { partialling shared variance }\end{array}$ & $\begin{array}{l}\text { Residual } r \text { with age after } \\
\text { partialling shared variance }\end{array}$ \\
\hline \multicolumn{3}{|l|}{ Fluid cognition } \\
\hline Speed & $-0.555^{* *}(0.308)$ & $-0.130(0.017)$ \\
\hline Executive function & $-0.610^{* *}(0.372)$ & $-0.235^{*}(0.055)$ \\
\hline Memory & $-0.449^{* *}(0.202)$ & $-0.125(0.016)$ \\
\hline \multicolumn{3}{|c|}{ White-matter integrity } \\
\hline Sensorimotor FA & $-0.540^{* *}(0.292)$ & $-0.100(0.010)$ \\
\hline Frontoparietal FA & $-0.654^{* *}(0.428)$ & $-0.382^{* *}(0.146)$ \\
\hline \multicolumn{3}{|l|}{ Resting-state FC } \\
\hline $\begin{array}{l}\text { Default mode } \\
\text { resting-state FC }\end{array}$ & $-0.484^{* *}(0.234)$ & $-0.150(0.022)$ \\
\hline $\begin{array}{l}\text { Sensorimotor } \\
\text { resting-state FC }\end{array}$ & $-0.619^{* *}(0.383)$ & $-0.418^{* *}(0.175)$ \\
\hline $\begin{array}{l}\text { Frontoparietal } \\
\text { resting-state FC }\end{array}$ & $-0.359^{* *}(0.129)$ & $-0.078(0.006)$ \\
\hline \multicolumn{3}{|c|}{$\begin{array}{l}\text { White-matter } \\
\text { hyperintensity volume }\end{array}$} \\
\hline PV & $0.496^{* *}(0.246)$ & $0.425^{* *}(0.181)$ \\
\hline DWM & $0.254^{*}(0.065)$ & $-0.013(0.0002)$ \\
\hline
\end{tabular}

Note. Values are $r$, with $r^{2}$ in parentheses.

${ }^{*} p<0.01$.

${ }^{* * *} p<0.001$.

Key: DWM, deep white matter hyperintensity volume; FA, fractional anisotropy; FC, functional connectivity; PV, periventricular white-matter hyperintensity volume.

Further, the sensorimotor resting-state functional connectivity variable exhibited a significant relation to residual executive function ( $b$ path) independently of age. This latter relation was positive, indicating that increased functional connectivity was associated with higher executive function. The total effect of age on residual executive function (i.e., before including the mediating effects) was significant, and the direct effect (i.e., after including the mediating effects) was not significant, implying complete mediation by sensorimotor resting-state functional connectivity. The mediating effect of sensorimotor resting-state functional connectivity appears to depend on having a wide range of adult age, as the corresponding effect was not significant when the analysis was limited to 46 participants 60 years of age and older (Supplementary Material, Table S2).

For each of the residual cognitive measures, tests of the age $x$ mediator interactions (i.e., moderation) did not yield any significant effects.

Although sensorimotor resting-state functional connectivity is a regionally specific imaging variable, it comprises 3 indicator variables from the ICA analysis. We thus constructed an additional mediation model with these 3 variables, ICA components for motor, primary visual, and basal ganglia/thalamus networks, as parallel mediators of the relation between age and residual executive function. The results (Table 6) indicated significant age-related decline ( $a$ paths) for primary visual and basal ganglia/thalamus network connectivity. But none of the variables exhibited either an age-independent relation to residual executive function ( $b$ paths) or a mediating influence on the age-executive function relation. However, the total effect of age ( $c$ path) was significant whereas the direct effect of age ( $c^{\prime}$ path) was not. This pattern implies that the mediation associated with the sensorimotor factor score (Fig. 2) reflects the combined contribution of all 3 indicator variables rather than 1 or 2 of them individually.

\section{Discussion}

These results illustrate the way in which models of age-related differences in brain imaging and cognitive measures vary with the level of analysis. At a general level of analysis, we defined fluid cognition by the first unrotated factor from 9 psychometric and RT tests, and in a similar manner defined each of 3 imaging modalities of white-matter integrity, resting-state connectivity, and $\mathrm{WMH}$ volume, from the first factor of several individual imaging measures. As we hypothesized, age exhibited strong, independent effects on these general cognitive and imaging measures. Consistent with the pattern reported by Salthouse et al. (2015), age was associated both with decline in fluid cognition, independent of the imaging variables, and with decline in the imaging measures, independent of cognition. The mediation analyses, at this general level, were also consistent with the independent age-effects model. None of the summary measures from the imaging modalities exerted either an independent influence on fluid cognition, when controlled for age, or a mediating influence on the relation between age and fluid cognition (Table 3 ).

At a more specific level of analysis, we distinguished 3 domains of fluid cognition and incorporated anatomically defined components of the brain imaging measures. In our construction of specific summary measures, we continued to use a factor-analytic approach for defining both the cognitive and imaging measures, using the first unrotated factor from several individual measures within each domain of interest. Thus, although our outcome variables exhibited additional specificity, they were also latent variables that each comprised several individual tests. To control for the shared variance among these individual measures, we partialled each unrotated factor for the set of variables not included in the factor.

Within each of the cognitive and imaging domains, one of the summary measures exhibited age-related decline, beyond the shared variance (Table 4). Among the cognitive variables, executive function was most vulnerable to age-related decline, in line with previous behavioral studies (Verhaeghen and Cerella, 2002; Wecker et al., 2000). The age-related decline in frontoparietal FA is consistent with previous DTI studies (Bennett and Madden, 2014; Carmichael and Lockhart, 2012; Madden et al., 2012; Sullivan and Pfefferbaum, 2011), and the age-related increase in PV WMH volume confirms the role of these WMHs in disrupting the long whitematter tracts connecting cortical association regions (Smith et al., 2011; Soderlund et al., 2006; van den Heuvel et al., 2006).

For both the general and domain-specific cognitive and imaging measures, the age-related declines were relatively linear from 19 to 79 years of age (Fig. 2 and Table 4), and we did not detect any agerelated moderation of the variables tested as potential mediators. Salthouse $(2004,2009)$ has reported that age-related decline in fluid cognition is primarily linear and has proposed that this pattern represents a continuous process that begins early in adulthood. The present results suggest that white-matter integrity, resting-state connectivity, and $\mathrm{WMH}$ volume also exhibit a linear age-related pattern, consistent with the cognitive data, suggesting that the cognitive and imaging measures reflect different aspects of the underlying influence of age on central nervous system functioning (Baltes and Lindenberger, 1997; Birren, 1965; Madden, 2001). Nonlinear age-related trends, typically in the form of accelerated decline at later stages of adulthood, have been observed for structural imaging measures of gray and white matter (Fjell and Walhovd, 2010) and for resting-state functional connectivity (Chan et al., 2014). The present age ceiling of 79 years and sample size may have limited the power to detect nonlinear age-related trends, and further research on the exact shape of the age-related trends for these imaging measures is warranted.

The stronger age-related decline in sensorimotor resting-state functional connectivity (comprising visual, motor, and basal ganglia/thalamus networks) is surprising, because the default mode network has been more widely discussed as a target of age-related decline (Andrews-Hanna et al., 2007; Biswal et al., 2010; Ferreira and Busatto, 2013). However, several reports of lowered 
Table 5

Regionally specific imaging variables as mediators of age relation to general fluid cognition, residual speed, residual executive function, and residual memory

\begin{tabular}{|c|c|c|c|c|c|c|c|}
\hline Model & Effect & $S E$ & $\beta$ & $t$ & $p$ & Lower CI & Upper CI \\
\hline \multicolumn{8}{|l|}{$x \rightarrow m$ ( $a$ path; for all models) } \\
\hline Age $\rightarrow$ Sensorimotor FA & -0.0286 & 0.0038 & -0.5368 & -7.5788 & 0.0001 & -0.0385 & $-\mathbf{0 . 0 1 8 7}$ \\
\hline Age $\rightarrow$ frontoparietal FA & -0.0356 & 0.0035 & -0.6501 & $-\mathbf{1 0 . 2 5 3 1}$ & 0.0001 & -0.0446 & -0.0265 \\
\hline Age $\rightarrow$ default mode RS FC & -0.0177 & 0.0027 & -0.4838 & -6.6051 & 0.0001 & -0.0247 & -0.0107 \\
\hline Age $\rightarrow$ sensorimotor RS FC & $-\mathbf{0 . 0 1 7 1}$ & 0.0018 & -0.6192 & -9.6995 & 0.0001 & $-\mathbf{0 . 0 2 1 7}$ & -0.0125 \\
\hline Age $\rightarrow$ frontoparietal RS FC & -0.0129 & 0.0028 & $-\mathbf{0 . 3 5 8 8}$ & -4.5258 & 0.0001 & -0.0203 & -0.0054 \\
\hline Age $\rightarrow$ PV WMH volume & 0.0237 & 0.0035 & 0.4960 & 6.6895 & 0.0001 & 0.0144 & 0.0329 \\
\hline Age $\rightarrow$ DWM WMH volume & 0.0109 & 0.0036 & 0.2545 & 3.0500 & 0.0027 & 0.0016 & 0.0203 \\
\hline
\end{tabular}

Model 1 (general fluid cognition): $\mathrm{x}=$ age; $\mathrm{m}=$ sensorimotor FA; frontoparietal FA; default mode FC; sensorimotor resting-state FC; frontoparietal

resting-state FC; PV WMH volume; DWM WMH volume; $y=$ general fluid cognition

Model $1 m \rightarrow y$ ( $b$ path)

Sensorimotor FA $\rightarrow$ Gen Fluid Cogn

Frontoparietal FA $\rightarrow$ Gen Fluid Cogn

Default mode FC $\rightarrow$ Gen Fluid Cogn

Sensorimotor RS FC $\rightarrow$ Gen Fluid Cogn

Frontoparietal RS FC $\rightarrow$ Gen Fluid Cogn

PV WMH volume $\rightarrow$ Gen Fluid Cogn

DWM WMH volume $\rightarrow$ Gen Fluid Cogn

Model 1 mediation effects ( $a \times b$ interaction)

Sensorimotor FA

Frontoparietal FA

Default mode RS FC

Sensorimotor RS FC

Frontoparietal RS FC

PV WMH volume

DWM WMH volume

Total effect for age ( $c$ path)

$\begin{array}{rr}0.0228 & 0.0807 \\ -0.0324 & 0.0916 \\ -0.0529 & 0.1084 \\ 0.0691 & 0.1459 \\ 0.1978 & 0.1028 \\ -0.1249 & 0.0946 \\ 0.1557 & 0.0915 \\ & \\ -0.0007 & 0.0025 \\ 0.0012 & 0.0034 \\ 0.0009 & 0.0019 \\ -0.0012 & 0.0025 \\ -0.0025 & 0.0013 \\ -0.0030 & 0.0024 \\ 0.0017 & 0.0013 \\ -\mathbf{0 . 0 3 4 7} & \mathbf{0 . 0 0 3 0} \\ -\mathbf{0 . 0 3 1 1} & \mathbf{0 . 0 0 5 0}\end{array}$

$\begin{array}{rr}0.0199 & 0.2821 \\ -0.0179 & -0.3540 \\ -0.0342 & -0.4883 \\ 0.0525 & 0.4735 \\ 0.1338 & 1.9237 \\ -0.1022 & -1.3205 \\ 0.1265 & 1.7014 \\ & \\ -0.0107 & - \\ 0.0116 & - \\ 0.0165 & - \\ -0.0325 & - \\ -0.0480 & - \\ -0.0507 & - \\ 0.0322 & - \\ -\mathbf{0 . 6 9 8 1} & -\mathbf{1 1 . 6 0 0 6} \\ -\mathbf{0 . 6 1 6 6} & -\mathbf{6 . 2 8 8 9}\end{array}$

\begin{tabular}{ll}
0.7783 & -0.1368 \\
0.7239 & -0.2135 \\
0.6262 & -0.2674 \\
0.6366 & -0.2195 \\
0.0565 & -0.0056 \\
0.1890 & -0.3120 \\
0.0912 & -0.0253 \\
- & \\
- & -0.0055 \\
- & -0.0056 \\
- & -0.0026 \\
- & -0.0060 \\
- & -0.0064 \\
- & -0.0081 \\
$\mathbf{0 . 0 0 0 1}$ & -0.0002 \\
$\mathbf{0 . 0 0 0 1}$ & $-\mathbf{0 . 0 4 0 6}$ \\
\hline
\end{tabular}

0.1823

0.1487

0.1615

0.3577

0.4013

0.0622

0.3367

0.0042

0.0078

0.0049

0.0040

0.0009

0.0013

0.0048

Direct effect for age ( $c^{\prime}$ path)

$0.0050-\mathbf{0 . 6 1 6 6}$

0.0001

$-0.0409$

$\mathbf{0 . 0 2 8 8}$

Model 2 (residual speed): $\mathrm{x}$ = age; $\mathrm{m}=$ sensorimotor FA; frontoparietal FA; default mode FC; sensorimotor resting-state FC; frontoparietal resting-state FC;

PV WMH volume; DWM WMH volume; $\mathrm{y}=$ residual speed

Model $2 m \rightarrow y$ ( $b$ path)

Frontoparietal $\mathrm{FA} \rightarrow$ residual $\mathrm{Spe}$

Default mode RS FC $\rightarrow$ Residual speed

0.0499

\subsection{2}

0.0846

\subsection{4}

0.4905

$-0.1388$

$-0.2708$

0.2386

-0.0566
0.0894

0.0820
0.0970

-0.0764
0.1115

-0.6905
0.9209

0.4911

$-0.1247$

$-1.3549$

0.3588

-0.1769
0.0569

0.1306

Frontoparietal RS FC $\rightarrow$ Residual speed

0.0569
-0.0181

0.0539

0.6179

0.1778

$-0.1643$

0.1576

PV WMH volume $\rightarrow$ residual speed

0.0921
0.0847

$-0.0312$

$-0.2137$

0.5377

$-0.5182$

$-0.1837$

$-0.2393$

0.1644

DWM WMH volume $\rightarrow$ Residual speed

Model 2 mediation effects ( $a \times b$ interaction)

Sensorimotor FA

Frontoparietal FA

Default mode RS FC

Sensorimotor RS FC

Frontoparietal RS FC

PV WMH volume

DWM WMH volume

$-0.0014$

0.0819

0.0491

0.7016

0.2975
0.2032

0.2455

\section{Total effect for age (c path)}

$\begin{array}{ll}-0.0014 & 0.0019\end{array}$

$-0.0454$

0.0497

$-0.0016$

0.0031

0.0030

0.0018

$-0.0539$

0.0772

$-0.0007$

0.0021

$-0.0004$

0.0013

0.0003

0.0021

$-0.0193$

$-0.0155$

0.0125

$-0.0046$

0.0008
0.0027

0.0044

$-0.1296$

0.3840

$-$

$-0.0066$

$-0.0060$

$-0.0069$

0.0034

$-$

-

$-$

Direct effect for age (c' path)

$-0.1349$

$-1.7430$

$-0.0026$

$-0.0039$

$-0.0063$

$-0.0019$

$-0.0116$

$-0.0174$

0.0099

0.0029

0.0093

0.0033

0.0053

0.0030

0.0023

0.0057

Model 3 (residual executive function): $\mathrm{x}=$ age; $\mathrm{m}=$ sensorimotor FA; frontoparietal FA; default mode FC; sensorimotor resting-state FC; frontoparietal

resting-state FC; PV WMH volume; DWM WMH volume; y = residual executive function

Model $3 m \rightarrow y$ (b path)

Sensorimotor $\mathrm{FA} \rightarrow$ residual executive

Frontoparietal FA $\rightarrow$ residual executive

Default mode RS FC $\rightarrow$ residual executive

ensorimotor RS FC $\rightarrow$ Residual executive

$-0.0495$

$-0.0097$

0.0581

$-0.0952$

$-0.0785$

0.2844

0.0660

$-0.0233$

$-0.8522$

$-0.1472$

0.3956

$-0.2015$

0.1024

Frontoparietal RS FC $\rightarrow$ residual executive

0.0397

0.0781

$-0.1131$

$-1.0040$

0.8832

$-0.1822$

0.1628

PV WMH volume $\rightarrow$ residual executive

DWM WMH volume $\rightarrow$ residual executive

Model 3 mediation effects ( $a \times b$ interaction)

Sensorimotor FA

$-0.0846$

0.0741

0.0553

2.7049

0.3172

$-0.2827$

0.1258

0.5356

0.5931

0.0096

0.5592

-0.1405
0.1590

-1.2417
1.5784

0.2166

$-0.1540$

0.2334

0.0682
0.0660

0.1590

0.1169

$-0.0683$

0.0935
0.2765

\subsection{4}

Pefaultiont

0.0003

0.0014

$\mathbf{- 0 . 0 0 4 9}$

0.0017

0.0511

0.0023

Sensorimotor resting-state FC

Frontoparietal RS FC

PV WMH volume

DWM WMH volume

$-0.0005$

$-0.0020$

0.0011

$-0.0061$

Direct effect for age (c' path)

$-0.0030$

0.0016

0.0018

0.0010

0.0017

0.0008

0.0022

0.0036

0.0151
0.0547
$-\mathbf{0 . 1 7 4 4}$
-0.0198
-0.0697
0.0405
$-\mathbf{0 . 2 3 3 3}$
-0.1309

-
-
-
-
-
-
-
-2.7924
-0.8495

-
-
-
-
-
-
$\mathbf{0 . 0}$

$-0.0030$

0.0061

0.0071

0.0058

$-0.0027$

$\mathbf{- 0 . 0 0 9 8}$

$-0.0037$

0.0020

0.0022

0.0038

$\mathbf{- 0 . 0 0 0 4}$

Model 4 (residual memory): $x=$ age $m=$ sensorimotor FA; frontoparietal FA; default mode FC; sensorimotor resting-state FC; frontoparietal resting-state FC;

PV WMH volume; DWM WMH volume; $y=$ residual memory

Model $4 m \rightarrow y$ (b path)

Sensorimotor FA $\rightarrow$ residual memory

Frontoparietal FA $\rightarrow$ residual memory 
Table 5 (continued)

\begin{tabular}{|c|c|c|c|c|c|c|c|}
\hline Model & Effect & $S E$ & $\beta$ & $t$ & $p$ & Lower CI & Upper CI \\
\hline Default mode FC $\rightarrow$ residual memory & -0.0436 & 0.0771 & -0.0606 & -0.5649 & 0.5731 & -0.2451 & 0.1580 \\
\hline Sensorimotor RS FC $\rightarrow$ residual memory & -0.0477 & 0.1038 & -0.0448 & -0.4598 & 0.6464 & -0.3189 & 0.2235 \\
\hline Frontoparietal RS FC $\rightarrow$ residual memory & 0.0172 & 0.0732 & 0.0232 & 0.2355 & 0.8142 & -0.1740 & 0.2084 \\
\hline PV WMH volume $\rightarrow$ residual memory & 0.0376 & 0.0673 & 0.0708 & 0.5590 & 0.5771 & -0.1382 & 0.2134 \\
\hline DWM WMH volume $\rightarrow$ residual memory & -0.0442 & 0.0651 & -0.0744 & -0.6792 & 0.4982 & -0.2143 & 0.1259 \\
\hline \multicolumn{8}{|l|}{ Model 4 mediation effects ( $a \times b$ interaction) } \\
\hline Sensorimotor FA & 0.0016 & 0.0017 & 0.0637 & - & - & -0.0029 & 0.0062 \\
\hline Frontoparietal FA & -0.0028 & 0.0022 & -0.1134 & - & - & -0.0084 & 0.0029 \\
\hline Default mode RS FC & 0.0008 & 0.0016 & 0.0293 & - & - & -0.0030 & 0.0053 \\
\hline Sensorimotor RS FC & 0.0008 & 0.0017 & 0.0277 & - & - & -0.0036 & 0.0055 \\
\hline Frontoparietal RS FC & -0.0002 & 0.0009 & -0.0083 & - & - & -0.0028 & 0.0020 \\
\hline PV WMH volume & 0.0009 & 0.0018 & 0.0351 & - & - & -0.0039 & 0.0055 \\
\hline DWM WMH volume & -0.0005 & 0.0008 & -0.0189 & - & - & -0.0028 & 0.0016 \\
\hline Total effect for age ( $c$ path) & -0.0032 & 0.0021 & -0.1240 & -1.5210 & 0.1305 & -0.0087 & 0.0023 \\
\hline Direct effect for age ( $c^{\prime}$ path) & -0.0038 & 0.0035 & -0.1393 & -1.0729 & 0.2853 & -0.0130 & 0.0054 \\
\hline
\end{tabular}

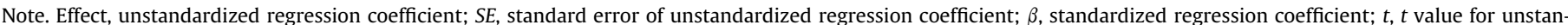

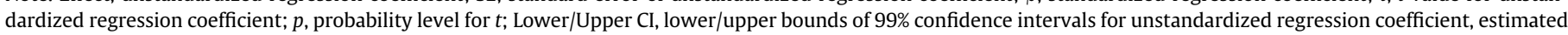
from bootstrap sampling with 10,000 samples.

Significant effects are presented in bold.

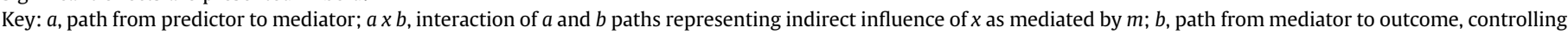

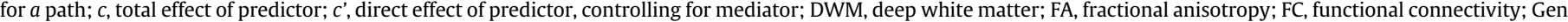
Fluid Cogn, general fluid cognition; $m$, mediator; PV, periventricular; RS, resting state; WMH, white-matter hyperintensity; $x$, predictor variable; $y$, outcome variable.

resting-state functional connectivity within older adults' visual networks have also emerged (Betzel et al., 2014; Onoda et al., 2012; Yan et al., 2011; Zhang et al., 2014). Similarly, several studies of both structural and functional connectivity indicate significant agerelated decline in networks associated with basal ganglia and thalamus (Fama and Sullivan, 2015; Kievit et al., 2014; Ystad et al., 2011).

In our analyses of these more specific cognitive and imaging variables, the independent age-effects model did not hold. The 3 residual imaging measures that were age-sensitive (frontoparietal FA, sensorimotor resting-state functional connectivity, and PV WMH volume) continued to exhibit significant age-related decline when partialled for the 3 cognitive domains, but the age-related difference in residual executive function was no longer significant when partialled for the regionally specific imaging measures (see results, specific cognitive and imaging variables). Thus, age did not have a specific relation to executive function that was independent

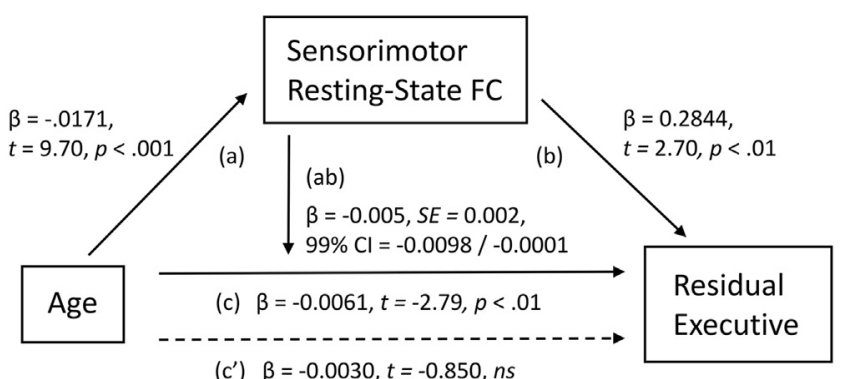

Fig. 3. Mediation of the relation between age and residual executive function by sensorimotor resting-state functional connectivity. Results indicated that the path $(a)$ from the predictor (age) to the mediator (sensorimotor resting-state functional connectivity) was significant, as was the path $(b)$ from sensorimotor resting-state functional connectivity to the outcome variable (residual executive function). The interaction of the $a$ and $b$ paths was significant, indicating that age exerted an indirect influence on residual executive function, through sensorimotor resting-state functional connectivity. The model included the other 6 regionally specific imaging measures as mediators; the mediation effect is covaried for these other measures (Table 5, model 3). Significant paths are presented as solid lines; nonsignificant paths are presented as dotted lines. Path $(c)$ is the total effect of the relation between the predictor and outcome variables, and path $\left(c^{\prime}\right)$ is the direct effect of the predictor, taking into account the effect of the mediator. Unstandardized regression coefficients $(\beta)$ and $99 \%$ confidence intervals were estimated from 10,000 percentile-based bootstrap samples. Abbreviations: $\mathrm{CI}, 99 \%$ confidence intervals; FC, functional connectivity. of the imaging measures. The mediation analyses (Table 5) provided additional information on this point by showing that age influenced the residual executive function measure indirectly, through restingstate functional connectivity in sensorimotor networks. Age led to significant decline in the factor score representing this group of networks (the $a$ path in Fig. 3), but the sensorimotor networks also exhibited a positive relation to executive function (i.e., higher connectivity associated with better executive function), independently of age (the $b$ path in Fig. 3). The total effect of age on executive function (the $c$ path in Fig. 3) was significant, but the direct effect (the c' path in Fig. 3) was not, implying that sensorimotor resting-state functional connectivity completely mediated the ageexecutive function relation. This mediation effect, however, was not attributable to any of the 3 networks of the sensorimotor functional connectivity measure (primary visual, motor cortex, and basal ganglia/thalamus), but rather to their combined effects (Table 6).

Although we had predicted that mediation of age-cognitive relations would be more clearly evident with regionally defined imaging measures (Gazes et al., 2016; Lee et al., 2016), this particular pattern of mediation, involving sensorimotor resting-state functional connectivity, was unexpected. Hedden et al. (2016), and Fjell et al. (2016) both reported that measures of structural connectivity (e.g., white matter FA or volume) were stronger mediators of agerelated variance in fluid cognition than resting-state functional connectivity. Similarly, from previous evidence linking frontoparietal regions to age-related decline in fluid cognition (Bennett and Madden, 2014; Gold et al., 2010; Kennedy and Raz, 2009; Monge et al., 2016; Shaw et al., 2015), we had expected that frontoparietal FA or connectivity would mediate the age-cognition relation. Only sensorimotor resting-state functional connectivity, however, emerged as a mediator of age-related decline in executive function (Table 6 and Fig. 3). The sensorimotor network and executive function measures both exhibited strong age-related effects, relative to other measures in their respective domains (Table 4), which may have contributed to the mediating effect for sensorimotor resting-state functional connectivity. However, frontoparietal FA and PV WMH volume also exhibited significant age-related variance when partialled for the other domain-relevant imaging measures (Table 4), and yet neither of these measures was a significant mediator.

As noted previously in this discussion, evidence is accumulating to suggest an important role for structural and functional 
Table 6

Sensorimotor resting-state FC indicator variables as mediators of age-residual executive function relation

\begin{tabular}{|c|c|c|c|c|c|c|c|}
\hline Model & Effect & $S E$ & $\beta$ & $t$ & $p$ & Lower CI & Upper CI \\
\hline \multicolumn{8}{|c|}{$\begin{array}{l}\text { Model: } x=\text { age; } m=\text { primary visual RS FC; motor cortex RS FC; basal ganglia/thalamus RS FC; } y=\text { residual executive function } \\
\quad x \rightarrow m \text { (a path) }\end{array}$} \\
\hline Age $\rightarrow$ primary visual RS FC & $-\mathbf{0 . 0 3 3 8}$ & 0.0054 & -0.4570 & -6.2379 & 0.0001 & -0.0480 & $-\mathbf{0 . 0 1 9 7}$ \\
\hline Age $\rightarrow$ motor cortex RS FC & 0.0040 & 0.0043 & 0.0897 & 0.9464 & 0.3456 & -0.0071 & 0.0152 \\
\hline Age $\rightarrow$ basal ganglia/thalamus RS FC & -0.0402 & 0.0054 & -0.5231 & -7.5048 & 0.0001 & -0.0542 & -0.0262 \\
\hline \multicolumn{8}{|l|}{$m \rightarrow y(b$ path $)$} \\
\hline Primary visual RS FC $\rightarrow$ residual executive & 0.0457 & 0.0335 & 0.1235 & 1.3637 & 0.1749 & -0.0418 & 0.1333 \\
\hline Motor cortex RS FC $\rightarrow$ residual executive & -0.0349 & 0.0427 & -0.0679 & -0.8163 & 0.4157 & -0.1464 & 0.0767 \\
\hline Basal ganglia/thalamus RS FC $\rightarrow$ residual executive & 0.0675 & 0.0340 & 0.1898 & 1.9837 & 0.0493 & -0.0214 & 0.1563 \\
\hline \multicolumn{8}{|l|}{ Mediation effects ( $a \times b$ interaction) } \\
\hline Primary visual RS FC & -0.0015 & 0.0012 & -0.0564 & - & - & -0.0046 & 0.0019 \\
\hline Motor cortex RS FC & -0.0001 & 0.0003 & -0.0061 & - & - & -0.0015 & 0.0006 \\
\hline Basal ganglia/thalamus RS FC & -0.0027 & 0.0014 & -0.0993 & - & - & -0.0067 & 0.0009 \\
\hline Total effect for age ( $c$ path) & -0.0062 & 0.0022 & -0.2333 & -2.8551 & 0.0050 & -0.0119 & -0.0005 \\
\hline Direct effect for age ( $c^{\prime}$ path) & -0.0018 & 0.0028 & -0.0716 & -0.6409 & 0.5227 & -0.0092 & 0.0055 \\
\hline
\end{tabular}

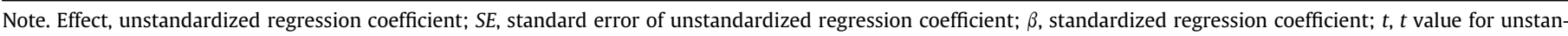

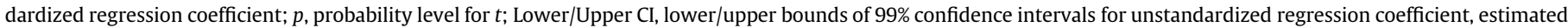
from bootstrap sampling with 10,000 samples.

Significant effects are presented in bold.

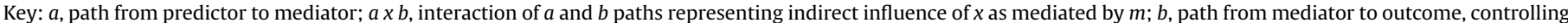

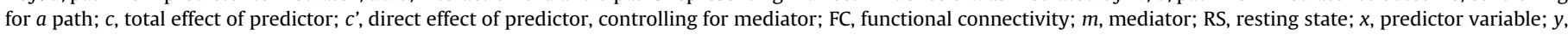
outcome variable.

connectivity of subcortical structures in neurocognitive aging (Fama and Sullivan, 2015; Kievit et al., 2014; Madden et al., 2004; Samanez-Larkin et al., 2012; Ystad et al., 2011). In their 1970 review, Hicks and Birren (1970) concluded, from studies of human neurodegenerative disease and animal neurophysiology, that the basal ganglia and their associated cortical targets comprised a neural mechanism of age-related psychomotor slowing. Direct comparison of the present results with previous mediation analyses (Fjell et al., 2016; Hedden et al., 2016; Kievit et al., 2014; Shaw et al., 2015 ) is difficult in view of the different sets of variables chosen as mediators, and as outcome variables, across studies. The present results underscore the importance of resting-state functional connectivity, within a regionally specific network, as a potential source of age-related decline in executive function (Shaw et al., 2015).

These findings are constrained by several limitations. First, we grouped the indicator variables for the specific cognitive and imaging domains on an a priori basis, from the previous literature and known anatomy. A more empirically derived grouping of the indicator variables (e.g., from factor analysis) would lead to a different set of summary measures and to different models of the age-related effects. Second, our interest was in imaging measures related to structural and functional connectivity, and other measures, particularly cortical thickness, white-matter volume, amyloid burden, and cerebral metabolic rates, are relevant as potential mediators (Fjell et al., 2016; Hedden et al., 2016; Salthouse et al., 2015). The structural imaging variables, in particular, may have additional predictive value when the sample is not as highly select in cognitive and physical health as in the present case. Third, the mediation effect that we observed, for sensorimotor resting-state functional connectivity, may be related to sampling a relatively wide age range from 19 to 79 years, as the mediation effect was no longer significant when limited to individuals 60 years of age and older. Finally, caution is needed in the interpretation of agerelated effects in cross-sectional data. It is important to emphasize that age-related differences in cognitive and neural measures, as reported here, do not directly represent longitudinal change in those measures (Hofer and Sliwinski, 2001; Lindenberger et al., 2011). Longitudinal designs, however, although valuable for including information regarding change over time, also have limitations. For example, as a result of the correlation of scores across measurement occasions, the systematic variance in change over time may be small relative to the variance at each measurement occasion (Salthouse, 2011a; Salthouse and Nesselroade, 2002). Thus, converging evidence from both cross-sectional and longitudinal analyses would enhance the interpretation of these findings.

\section{Conclusions}

Models of neural disconnection in age-related cognitive decline vary across different levels of analysis. At a broad level, with a single measure of fluid cognition and summary measures of cerebral white-matter integrity, resting-state functional connectivity, and WMH volume, age had a strong and independent influence on the cognitive and imaging measures, and the imaging variables did not influence the age-cognition relation. At a more specific level of analysis, with individual measures of fluid cognition and regionally defined imaging variables, and controlling for the shared variance among indicator variables, we obtained evidence for individual sources of disconnection. Executive function exhibited age-related decline that was independent of perceptual speed and memory. Age, however, influenced executive function indirectly, through a decline in the resting-state functional connectivity of sensorimotor networks (visual, motor, and basal ganglia/thalamus). Each of our regionally specific imaging measures, while representing anatomically plausible domains, still comprised several indicator variables with potentially distinct contributions. In future investigations, it would be useful to explore additional models at a more specific level, testing the roles of the individual indicator variables as sources of disconnection in neurocognitive aging.

\section{Disclosure statement}

The authors have no conflicts of interest to disclose.

\section{Acknowledgements}

This research was supported by NIH research grant R01 AG039684. The authors are grateful to Ling Zou, Syam Gadde, Chris Petty, Max Horowitz, and Kristin Sundy for their assistance. 


\section{Appendix A. Supplementary data}

Supplementary data associated with this article can be found, in the online version, at http://dx.doi.org/10.1016/j.neurobiolaging. 2017.01.027.

\section{References}

Andrews-Hanna, J.R., Snyder, A.Z., Vincent, J.L., Lustig, C., Head, D., Raichle, M.E., Buckner, R.L., 2007. Disruption of large-scale brain systems in advanced aging. Neuron 56, 924-935.

Bach, M., 1996. The Freiburg Visual Acuity test-automatic measurement of visual acuity. Optom. Vis. Sci. 73, 49-53.

Baltes, P.B., Lindenberger, U., 1997. Emergence of a powerful connection between sensory and cognitive functions across the adult life span: a new window to the study of cognitive aging? Psychol. Aging 12, 12-21.

Bartzokis, G., 2004. Age-related myelin breakdown: a developmental model of cognitive decline and Alzheimer's disease. Neurobiol. Aging 25, 5-18.

Basser, P.J., Pierpaoli, C., 1996. Microstructural and physiological features of tissues elucidated by quantitative-diffusion-tensor MRI. J. Magn. Reson. B. 111, 209-219.

Beaulieu, C., 2014. The biological basis of diffusion anisotropy. In: Johansen-Berg, H., Behrens, T.E.J. (Eds.), Diffusion MRI: From Quantitative Measurement to in Vivo Neuroanatomy, second ed. Elsevier, San Diego, CA, pp. 155-183.

Beck, A.T., 1978. The Beck Depression Inventory. Psychological Corporation, New York.

Beckmann, C.F., DeLuca, M., Devlin, J.T., Smith, S.M., 2005. Investigations into resting-state connectivity using independent component analysis. Philos. Trans. R. Soc. Lond. B Biol. Sci. 360, 1001-1013.

Bennett, I.J., Madden, D.J., 2014. Disconnected aging: cerebral white matter integrity and age-related differences in cognition. Neuroscience 276, 187-205.

Betzel, R.F., Byrge, L., He, Y., Goni, J., Zuo, X.-N., Sporns, O., 2014. Changes in structural and functional connectivity among resting-state networks across the human lifespan. NeuroImage 102, 345-357.

Birdsill, A.C., Koscik, R.L., Jonaitis, E.M., Johnson, S.C., Okonkwo, O.C., Hermann, B.P., Larue, A., Sager, M.A., Bendlin, B.B., 2014. Regional white matter hyperintensities: aging, Alzheimer's disease risk, and cognitive function. Neurobiol. Aging 35, 769-776.

Birren, J.E., 1965. Age changes in speed of behavior: its central nature and physiological correlates. In: Welford, A.T., Birren, J.E. (Eds.), Behavior, Aging, and the Nervous System. Thomas, Springfield, IL, pp. 191-216.

Bishop, N.A., Lu, T., Yankner, B.A., 2010. Neural mechanisms of ageing and cognitive decline. Nature 464, 529-535.

Biswal, B.B., Mennes, M., Zuo, X.N., Gohel, S, Kelly, C., Smith, S.M., Beckmann, C.F., Adelstein, J.S., Buckner, R.L., Colcombe, S., Dogonowski, A.M., Ernst, M., Fair, D., Hampson, M., Hoptman, M.J., Hyde, J.S., Kiviniemi, V.J., Kötter, R., Li, S.J., Lin, C.P., Lowe, M.J., Mackay, C., Madden, D.J., Madsen, K.H., Margulies, D.S., Mayberg, H.S., McMahon, K., Monk, C.S., Mostofsky, S.H., Nagel, B.J., Pekar, J.J., Peltier, S.J., Petersen, S.E., Riedl, V., Rombouts, S.A., Rypma, B., Schlaggar, B.L., Schmidt, S., Seidler, R.D., Siegle, G.J., Sorg, C., Teng, G.J., Veijola, J., Villringer, A., Walter, M., Wang, L., Weng, X.C., Whitfield-Gabrieli, S., Williamson, P., Windischberger, C., Zang, Y.F., Zhang, H.Y., Castellanos, F.X., Milham, M.P., 2010. Toward discovery science of human brain function. Proc. Natl. Acad. Sci. U. S. A. 107, 4734-4739.

Borghesani, P.R., Madhyastha, T.M., Aylward, E.H., Reiter, M.A., Swarny, B.R., Schaie, K.W., Willis, S.L., 2013. The association between higher order abilities, processing speed, and age are variably mediated by white matter integrity during typical aging. Neuropsychologia 51, 1435-1444.

Buckner, R.L., 2004. Memory and executive function in aging and AD: multiple factors that cause decline and reserve factors that compensate. Neuron 44 , $195-208$.

Calamante, F., Tournier, J.D., Jackson, G.D., Connelly, A., 2010. Track-density imaging (TDI): super-resolution white matter imaging using whole-brain track-density mapping. Neuroimage 53, 1233-1243.

Carmichael, O., Lockhart, S., 2012. The role of diffusion tensor imaging in the study of cognitive aging. Curr. Top Behav. Neurosci. 11, 289-320.

Catani, M., ffytche, D.H., 2005. The rises and falls of disconnection syndromes. Brain 128 (Pt 10), 2224-2239.

Chan, M.Y., Park, D.C., Savalia, N.K., Petersen, S.E., Wig, G.S., 2014. Decreased segregation of brain systems across the healthy adult lifespan. Proc. Nat. Acad. Sci. 111, E4997-E5006.

Charlton, R.A., Barrick, T.R. McIntyre, D. Shen, Y O'Sullivan, M., Howe, F.A. Clark, C.A., Morris, R.G., Markus, H.S., 2006. White matter damage on diffusion tensor imaging correlates with age-related cognitive decline. Neurology 66, 217-222.

Chen, G., Chen, G., Xie, C., Ward, B.D., Li, W., Antuono, P., Li, S.J., 2012. A method to determine the necessity for global signal regression in resting-state fMRI studies. Magn. Reson. Med. 68, 1828-1835.

Delis, D.C., Kramer, J., Kaplan, E., Ober, B.A., 1987. California verbal Learning Test (CVLT) Manual. Psychological Corporation, San Antonio (TX).
Dvorine, I., 1963. Dvorine Pseudo-isochromatic Plates, second ed. Harcourt, New York.

Fama, R. Sullivan, E.V., 2015. Thalamic structures and associated cognitive functions: relations with age and aging. Neurosci. Biobehav. Rev. 54, 29-37.

Ferreira, L.K., Busatto, G.F., 2013. Resting-state functional connectivity in norma brain aging. Neurosci. Biobehav. Rev. 37, 384-400.

Fjell, A.M., Sneve, M.H., Grydeland, H., Storsve, A.B., Walhovd, K.B., 2016. The disconnected brain and executive function decline in aging. Cereb. Cortex 27 2303-2317.

Fjell, A.M., Walhovd, K.B., 2010. Structural brain changes in aging: courses, causes and cognitive consequences. Rev. Neurosci. 21, 187-221.

Folstein, M.F., Folstein, S.E., McHugh, P.R., 1975. "Mini-mental state". A practical method for grading the cognitive state of patients for the clinician. J. Psychiatr. Res. 12, 189-198.

Gazes, Y., Bowman, F.D., Razlighi, Q.R., O'Shea, D., Stern, Y., Habeck, C., 2016. White matter tract covariance patterns predict age-declining cognitive abilities. Neuroimage $125,53-60$.

Geschwind, N., 1965a. Disconnexion syndromes in animals and man. I. Brain 88, 237-294.

Geschwind, N., 1965b. Disconnexion syndromes in animals and man. II. Brain 88, 585-644.

Gold, B.T., Powell, D.K., Xuan, L., Jicha, G.A., Smith, C.D., 2010. Age-related slowing of task switching is associated with decreased integrity of frontoparietal white matter. Neurobiol. Aging 31, 512-522.

Goodglass, H., Kaplan, E., 1972. Assessment of Aphasia and Related Disorders. Lea \& Febiger, Philadelphia.

Grady, C., 2012. The cognitive neuroscience of ageing. Nat. Rev. Neurosci. 13, 491-505.

Greenwood, P.M., 2000. The frontal aging hypothesis evaluated. J. Int. Neuropsychol Soc. 6, 705-726.

Greicius, M.D., Flores, B.H., Menon, V., Glover, G.H., Solvason, H.B., Kenna, H., Reiss, A.L., Schatzberg, A.F., 2007. Resting-state functional connectivity in major depression: abnormally increased contributions from subgenual cingulate cortex and thalamus. Biol. Psychiatry 62, 429-437.

Hayes, A.F., 2009. Beyond Baron and Kenny: statistical mediation analysis in the new millennium. Commun. Monogr. 76, 408-420.

Hayes, A.F., 2013. Introduction to Mediation, Moderation, and Conditional Process Analysis. Guilford, New York.

Hedden, T., Gabrieli, J.D., 2004. Insights into the ageing mind: a view from cognitive neuroscience. Nat. Rev. Neurosci. 5, 87-96.

Hedden, T., Schultz, A.P., Rieckmann, A., Mormino, E.C. Johnson, K.A., Sperling, R.A. Buckner, R.L., 2016. Multiple brain markers are linked to age-related variation in cognition. Cereb. Cortex 26, 1388-1400.

Hedden, T., Van Dijk, K.R., Shire, E.H., Sperling, R.A., Johnson, K.A., Buckner, R.L., 2012. Failure to modulate attentional control in advanced aging linked to white matter pathology. Cereb. Cortex 22, 1038-1051.

Hicks, L.H., Birren, J.E., 1970. Aging, brain damage, and psychomotor slowing. Psychol. Bull 74, 377-396.

Hofer, S.M., Sliwinski, M.J., 2001. Understanding ageing. An evaluation of research designs for assessing the interdependence of ageing-related changes. Gerontology 47, 341-352.

Howard, J.H., Howard, D.V., 2013. Aging mind and brain: is implicit learning spared in healthy aging? Front Psychol. 4, 817.

Jagust, W., 2013. Vulnerable neural systems and the borderland of brain aging and neurodegeneration. Neuron 77, 219-234.

Jones, D.K., Knösche, T.R., Turner, R., 2013. White matter integrity, fiber count, and other fallacies: the do's and don'ts of diffusion MRI. Neuroimage 73, 239-254.

Karama, S., Colom, R. Johnson, W., Deary, IJ., Haier, R., Waber, D.P., Lepage, C., Ganjavi, H., Jung, R., Evans, A.C.; Brain Development Cooperative Group, 2011. Cortical thickness correlates of specific cognitive performance accounted for by the general factor of intelligence in healthy children aged 6 to 18. NeuroImage $55,1443-1453$.

Kempton, M.J., Underwood, T.S., Brunton, S., Stylios, F., Schmechtig, A., Ettinger, U. Smith, M.S., Lovestone, S., Crum, W.R., Frangou, S., Williams, S.C., Simmons, A., 2011. A comprehensive testing protocol for MRI neuroanatomical segmentation techniques: evaluation of a novel lateral ventricle segmentation method. Neuroimage 58, 1051-1059.

Kennedy, K.M., Raz, N., 2009. Aging white matter and cognition: differential effects of regional variations in diffusion properties on memory, executive functions, and speed. Neuropsychologia 47, 916-927.

Kievit, R.A., Davis, S.W., Mitchell, D.J., Taylor, J.R., Duncan, J., Cam-CAN Research Team, Henson, R.N., Cam-CAN Research Team, 2014. Distinct aspects of frontal lobe structure mediate age-related differences in fluid intelligence and multitasking. Nat. Commun. 5, 5658.

Laird, A.R., Fox, P.M., Eickhoff, S.B., Turner, J.A., Ray, K.L., McKay, D.R., Glahn, D.C., Beckmann, C.F., Smith, S.M., Fox, P.T. 2011. Behavioral interpretations of intrinsic connectivity networks. J. Cogn. Neurosci. 23, 4022-4037.

Lee, S., Habeck, C., Razlighi, Q., Salthouse, T., Stern, Y., 2016. Selective association between cortical thickness and reference abilities in normal aging. Neuroimage $142,293-300$.

Lindenberger, U., von Oertzen, T., Ghisletta, P., Hertzog, C., 2011. Cross-sectional age variance extraction: what's change got to do with it? Psychol. Aging 26, 34-47.

Liu, Z., Wang, Y., Gerig, G., Gouttard, S., Tao, R., Fletcher, T., Styner, M., 2010. Quality control of diffusion weighted images. Proc. Soc. Photo Opt. In. 7628. 
Loonstra, A.S., Tarlow, A.R., Sellers, A.H., 2001. COWAT metanorms across age, education, and gender. Appl. Neuropsychol. 8, 161-166.

Mackinnon, D.P., Fairchild, A.J., 2009. Current directions in mediation analysis. Curr. Dir. Psychol. Sci. 18, 16.

Madden, D.J., 2001. Speed and timing of behavioral processes. In: Birren, J.E., Schaie, K.W. (Eds.), Handbook of the Psychology of Aging, fifth ed. Academic Press, San Diego, CA, pp. 288-312.

Madden, D.J., Bennett, I.J., Burzynska, A., Potter, G.G., Chen, N.K., Song, A.W., 2012. Diffusion tensor imaging of cerebral white matter integrity in cognitive aging. Biochim. Biophys. Acta 1822, 386-400.

Madden, D.J., Parks, E.L., 2017. Age differences in structural connectivity: diffusion tensor imaging and white matter hyperintensities. In: Cabeza, R., Nyberg, L., Park, D.C. (Eds.), Cognitive Neuroscience of Aging: Linking Cognitive and Cerebral Aging, second ed. Oxford, New York, pp. 71-103.

Madden, D.J., Whiting, W.L., Huettel, S.A., White, L.E., MacFall, J.R., Provenzale, J.M., 2004. Diffusion tensor imaging of adult age differences in cerebral white matter: relation to response time. Neuroimage 21, 1174-1181.

Maillard, P., Carmichael, O., Fletcher, E., Reed, B., Mungas, D., DeCarli, C., 2012. Coevolution of white matter hyperintensities and cognition in the elderly. Neurology 79, 442-448.

Monge, Z.A., Greenwood, P.M., Parasuraman, R., Strenziok, M., 2016. Individual differences in reasoning and visuospatial attention are associated with prefrontal and parietal white matter tracts in healthy older adults. Neuropsychology 30, 558-567.

Mori, S., Oishi, K., Jiang, H., Jiang, L., Li, X., Akhter, K., Hua, K., Faria, A.V., Mahmood, A., Woods, R., Toga, A.W., Pike, G.B., Neto, P.R., Evans, A., Zhang, J., Huang, H., Miller, M.I., van Zijl, P., Mazziotta, J., 2008. Stereotaxic white matter atlas based on diffusion tensor imaging in an ICBM template. Neuroimage 40, $570-582$.

O’Sullivan, M., Jones, D.K., Summers, P.E., Morris, R.G., Williams, S.C., Markus, H.S., 2001. Evidence for cortical "disconnection" as a mechanism of age-related cognitive decline. Neurology 57, 632-638.

Onoda, K., Ishihara, M., Yamaguchi, S., 2012. Decreased functional connectivity by aging is associated with cognitive decline. J. Cogn. Neurosci. 24, 2186-2198.

Park, D.C., Lautenschlager, G., Hedden, T., Davidson, N.S., Smith, A.D., Smith, P.K., 2002. Models of visuospatial and verbal memory across the adult life span. Psychol. Aging 17, 299-320.

Peters, A., 2002. The effects of normal aging on myelin and nerve fibers: a review. J. Neurocytol. 31, 581-593.

Power, J.D., Barnes, K.A., Snyder, A.Z., Schlaggar, B.L., Petersen, S.E., 2012. Spurious but systematic correlations in functional connectivity MRI networks arise from subject motion. Neuroimage 59, 2142-2154.

Raz, N., Yang, Y.Q., Rodrigue, K.M., Kennedy, K.M., Lindenberger, U., Ghisletta, P., 2012. White matter deterioration in 15 months: latent growth curve models in healthy adults. Neurobiol. Aging 33, e1-e5.

Rohlfing, T., 2013. Incorrect ICBM-DTI-81 atlas orientation and white matter labels. Front Neurosci. 7, 4.

Salat, D.H., 2011. The declining infrastructure of the aging brain. Brain Connect 1 , 279-293.

Salat, D.H., 2014a. Diffusion tensor imaging in the study of aging and age-associated neural disiease. In: Johansen-Berg, H., Behrens, T.E.J. (Eds.), Diffusion MRI: From Quantitative Measurement to In-vivo Neuroanatomy, second ed. Elsevier, San Diego, CA, pp. 257-281.

Salat, D.H., 2014b. Imaging small vessel-associated white matter changes in aging. Neuroscience 276, 174-186.

Salthouse, T.A., 1992. What do adult age differences in the Digit Symbol Substitution Test reflect? J. Gerontol. 47, P121-P128.

Salthouse, T.A., 1996. The processing-speed theory of adult age differences in cognition. Psychol. Rev. 103, 403-428.

Salthouse, T.A., 2004. What and when of cognitive aging. Curr. Dir. Psychol. Sci. 13, $140-144$.

Salthouse, T.A., 2009. When does age-related cognitive decline begin? Neurobiol. Aging 30, 507-514.

Salthouse, T.A., 2011a. All data collection and analysis methods have limitations: reply to Rabbitt (2011) and Raz and Lindenberger (2011). Psychol. Bull 137, 796-799.

Salthouse, T.A., 2011b. Neuroanatomical substrates of age-related cognitive decline. Psychol. Bull 137, 753-784.

Salthouse, T.A., 2017. Neural correlates of age-related slowing. In: Caebza, R, Nyberg, L., Park, D.C. (Eds.), Cognitive Neuroscience of Aging: Linking Cognitive and Cerebral Aging, second ed. Oxford, New York, pp. 259-272.

Salthouse, T.A., Habeck, C., Razlighi, Q., Barulli, D., Gazes, Y., Stern, Y., 2015. Breadth and age-dependency of relations between cortical thickness and cognition. Neurobiol. Aging 36, 3020-3028.
Salthouse, T.A., Nesselroade, J.R., 2002. An examination of the Hofer and Sliwinski evaluation. Gerontology 48, 18-21 discussion 2-9.

Samanez-Larkin, G.R., Levens, S.M., Perry, L.M., Dougherty, R.F., Knutson, B., 2012 Frontostriatal white matter integrity mediates adult age differences in probabilistic reward learning. J. Neurosci. 32, 5333-5337.

Sasson, E., Doniger, G.M., Pasternak, O., Tarrasch, R., Assaf, Y., 2013. White matter correlates of cognitive domains in normal aging with diffusion tensor imaging. Front Neurosci. 7, 32.

Saults, J.S., Cowan, N., 2007. A central capacity limit to the simultaneous storage of visual and auditory arrays in working memory. J. Exp. Psychol. Gen. 136, 663-684.

Schmidt, P., Gaser, C., Arsic, M., Buck, D., Forschler, A., Berthele, A., Hoshi, M., Ilg, R. Schmid, V.J., Zimmer, C., Hemmer, B., Mühlau, M., 2012. An automated tool for detection of FLAIR-hyperintense white-matter lesions in Multiple Sclerosis. Neuroimage 59, 3774-3783.

Seeley, W.W., Crawford, R.K., Zhou, J., Miller, B.L., Greicius, M.D., 2009. Neurodegenerative diseases target large-scale human brain networks. Neuron 62, 42-52.

Shaw, E.E., Schultz, A.P., Sperling, R.A., Hedden, T., 2015. Functional connectivity in multiple cortical networks is associated with performance across cognitive domains in older adults. Brain Connect 5, 505-516.

Shrout, P.E., Bolger, N., 2002. Mediation in experimental and nonexperimental studies: new procedures and recommendations. Psychol. Methods 7, 422-445.

Smith, E.E., Salat, D.H., Jeng, J., McCreary, C.R., Fischl, B., Schmahmann, J.D. Dickerson, B.C. Viswanathan, A., Albert, M.S., Blacker, D., Greenberg, S.M., 2011 Correlations between MRI white matter lesion location and executive function and episodic memory. Neurology 76, 1492-1499.

Smith, S.M., Brady, J.M., 1997. SUSAN-a new approach to low level image Processing. Int. J. Comput. Vis. 23, 45-78.

Smith, S.M., Jenkinson, M., Woolrich, M.W., Beckmann, C.F., Behrens, T.E., JohansenBerg, H., Bannister, P.R., De Luca, M., Drobnjak, I., Flitney, D.E., Niazy, R.K. Saunders, J., Vickers, J., Zhang, Y., De Stefano, N., Brady, J.M., Matthews, P.M., 2004. Advances in functional and structural MR image analysis and implementation as FSL. Neuroimage 23 (Suppl 1), S208-S219.

Soderlund, H., Nilsson, L.G., Berger, K., Breteler, M.M., Dufouil, C., Fuhrer, R. Giampaoli, S., Hofman, A., Pajak, A., de Ridder, M., Sans, S., Schmidt, R. Launer, L.J., 2006. Cerebral changes on MRI and cognitive function: the CASCADE study. Neurobiol. Aging 27, 16-23.

Stroop, J.R., 1935. Studies of interference in serial verbal reactions. J. Exp. Psychol. 18 643-662.

Sullivan, E.V., Pfefferbaum, A., 2011. Diffusion tensor imaging in aging and age-related neurodegenerative disorders. In: Jones, D.K. (Ed.), Diffusion MRI: Theory, Methods, and Applications. Oxford University Press, New York, pp. 624-643.

Tomasi, D., Volkow, N.D., 2012. Aging and functional brain networks. Mol. Psychiatry $17,549-558$

van den Heuvel, D.M., ten Dam, V.H., de Craen, A.J., Admiraal-Behloul, F., Olofsen, H. Bollen, E.L., Jolles, J., Murray, H.M., Blauw, G.J., Westendorp, R.G., van Buchem, M.A., 2006. Increase in periventricular white matter hyperintensities parallels decline in mental processing speed in a non-demented elderly population. J. Neurol. Neurosurg. Psychiatry 77, 149-153.

Verhaeghen, P., Cerella, J., 2002. Aging, executive control, and attention: a review of meta-analyses. Neurosci. Biobehav. Rev. 26, 849-857.

Walhovd, K.B., Westlye, L.T., Amlien, I., Espeseth, T., Reinvang, I., Raz, N., Agartz, I., Salat, D.H., Greve, D.N., Fischl, B., Dale, A.M., Fjell, A.M., 2011. Consistent neuroanatomical age-related volume differences across multiple samples. Neurobiol. Aging 32, 916-932.

Wechsler, D., 1997. Wechsler Adult Intelligence Scale-III. Psychological Corporation, New York.

Wecker, N.S., Kramer, J.H., Wisniewski, A., Delis, D.C., Kaplan, E., 2000. Age effects on executive ability. Neuropsychology 14, 409-414.

Yan, L., Zhuo, Y., Wang, B., Wang, D.J., 2011. Loss of coherence of low frequency fluctuations of BOLD fMRI in visual cortex of healthy aged subjects. Open Neuroimag. J. 5, 105-111.

Yeh, F.C., Verstynen, T.D., Wang, Y., Fernandez-Miranda, J.C., Tseng, W.Y., 2013. Deterministic diffusion fiber tracking improved by quantitative anisotropy. PLoS One 8, e80713.

Ystad, M., Hodneland, E., Adolfsdottir, S., Haasz, J., Lundervold, A.J., Eichele, T. Lundervold, A., 2011. Cortico-striatal connectivity and cognition in normal aging: a combined DTI and resting state fMRI study. Neuroimage 55, 24-31.

Zhang, H.-Y., Chen, W.-X., Jiao, Y., Xu, Y., Zhang, X.-R., Wu, J.-T., 2014. Selective vulnerability related to aging in large-scale resting brain networks. PLoS One 9 e108807.

Zhang, Y., Brady, M., Smith, S., 2001. Segmentation of brain MR images through a hidden Markov random field model and the expectation-maximization algorithm. IEEE Trans. Med. Imaging 20, 45-57. 